\title{
FLUORINATION EFFECTS ON THE INNER-SHELL SPECTRA OF UNSATURATED MOLECULES**
}

\author{
M.B. ROBIN \\ AT\&T Bell Laboratories, Murray Hill, NJ 07974 (U.S.A.) \\ I. ISHII, R. McLAREN and A.P. HITCHCOCK \\ Department of Chemistry, McMaster University, Hamilton, Ontario L8S 4M1 (Canada)
}

\begin{abstract}
The applicability of the perfluoro effect to the X-ray spectra $(300-800 \mathrm{eV})$ of unsaturated organic molecules is explored. The $\mathrm{C} 1 s$ and $\mathrm{F} 1 s$ (and $\mathrm{O} 1 s$ where appropriate) oscillator strength spectra of five fluoroethylenes, octafluorocyclopentene, formyl fluoride, carbonyl fluoride, hexafluorobutadiene, trifluoroacetic acid, hexafluorobutyne-2, hexafluoroacetone, and octafluoronaphthalene were derived from electron impact energy loss spectra recorded under electric-dipole scattering conditions. These spectra are analyzed and compared with those of their perhydro analogs, several of which (naphthalene, acetic acid, butyne-2) are reported for the first time. In unsaturated systems in which all the atoms lie in the molecular plane, such as ethylene, formaldehyde, benzene, etc., perfluorination results in approximately $10 \mathrm{eV}$ shifts of the inner-shell energy loss spectra to higher energies, yet the term values for the $\mathrm{C} 1 s \rightarrow 1 \pi^{*}$ excitations are shifted by only $1 \mathrm{eV}$ and often less. In direct contrast, the term values for the equivalent $\mathrm{C} 1 s \rightarrow 1 \pi^{*}$ excitations in unsaturated systems having atoms out of the molecular plane, such as butene-2 and acetone, are shifted upward by up to $3 \mathrm{eV}$ upon perfluorination. These different spectral behaviors of planar and nonplanar systems on fluorination quantitatively parallel those which were observed earlier for valence-level ionization potentials $(10-20 \mathrm{eV})$ and attributed to the perfluoro effect. It is observed for the first time that the $\mathrm{C} 1 s \rightarrow 1 \pi^{*}$ excitation energies in planar hydrocarbons are only very weakly dependent on the spatial extent of the $\pi$-electron system. An explanation involving a localized $\mathrm{C} 1 s$ hole is proposed to rationalize this behavior.

The perfluoro effect also predicts that excitations to $\sigma^{*}$ MO's will become relatively low-lying in highly fluorinated planar systems. Such low-lying inner-shell excitations induced by fluorination are observed in the fluoroethylene series and in the fluorocarbonyls. When the negative-ion spectra of the fluoroethylenes are assigned in a self-consistent manner, a $\sigma^{*} \mathrm{MO}$ is found to drop into the vicinity of $1 \pi^{*}$ upon fluorination. A similar intrusion of the lowest $\sigma^{*} \mathrm{MO}$ among the $\pi^{*}$ MO's is also observed upon fluorinating benzene, while evidence for this in the case of naphthalene is less clear, on account of the complex pattern of multiple $\mathrm{C} 1 s \rightarrow n \pi^{*}$ transitions in this molecule.

Inner-shell oscillator strength distributions are reported for all the spectra considered herein. In general, perfluorination increases the oscillator strengths of $\mathrm{C} 1 s \rightarrow 1 \pi^{*}$ transitions by up to a factor of two. Variation of the $\mathrm{C} 1 s \rightarrow 1 \pi^{*}$ and $\mathrm{O} 1 s \rightarrow 1 \pi^{*}$ oscillator strengths in the series $\mathrm{H}_{2} \mathrm{CO}$, $\mathrm{HFCO}, \mathrm{F}_{2} \mathrm{CO}$ shows clearly how the $1 \pi^{*} \mathrm{MO}$ becomes more polarized toward $\mathrm{C}$ as fluorination proceeds. In some cases, $\mathrm{C} 1 s \rightarrow \sigma^{*}(\mathrm{C}-\mathrm{F})$ oscillator strengths exceed those for $\mathrm{C} 1 s \rightarrow 1 \pi^{*}$ transitions.
\end{abstract}

\footnotetext{
**Submitted as part of the celebration of the 70th birthday of Professor Kai Siegbahn.
} 
The discrete excitations in the X-ray spectra of saturated molecules consist not only of Rydberg transitions converging upon the appropriate inner-shell ionization potentials, but also of valence excitations terminating at $\sigma^{*}$ MO's [1]. The latter orbitals may fall on either side of the ionization potential (the transition to $\sigma^{*}$ is called a shape resonance when it lies beyond the ionization potential ), and may be more or less mixed with levels of the Rydberg manifold depending upon symmetry, Rydberg penetration, and relative energies. Most often, the lowest Rydberg excitation lies below the lowest excitation to $\sigma^{*}$. When such saturated systems are oxidized so as to result in $\pi$-electron unsaturation, one or more new inner-shell excitations are introduced, terminating at the virtual $\pi^{*}$ MO's so formed. The lowest inner-shell excitation to $\pi^{*}$ usually falls below the lowest transitions to Rydberg or $\sigma^{*}$ MO's. The above generalizations on the inner-shell spectral patterns of saturated and unsaturated molecules also apply to excitations originating in the outer-shell levels. That is to say, the one-electron picture of virtual orbital ordering appears to be qualitatively valid.

Evidence is accumulating that the $1 \pi^{*}<$ Rydberg $<\sigma^{*}$ pattern of energy levels** stated above for unsaturated systems, and the Rydberg $<\sigma^{*}$ pattern for saturated systems can be severely upset when the molecule of interest is fluorinated. In particular, fluorination acts most directly on the $\sigma^{*}$ valence orbitals. There appear to be at least two reasons for these changes associated with fluorination:

(1) On replacing the $\mathrm{X}-\mathrm{H}$ bond by $\mathrm{X}-\mathrm{F}$, in some cases the bond strength is decreased drastically. For example, the bond strength decreases from $119 \mathrm{kcal}$ $\mathrm{mol}^{-1}$ to $38.4 \mathrm{kcal} \mathrm{mol}^{-1}$ on going from $\mathrm{H}_{2} \mathrm{O}$ to $\mathrm{F}_{2} \mathrm{O}$. As a consequence of the weak $\mathrm{O}-\mathrm{F}$ bond, there is a reduced $\sigma-\sigma^{*}$ splitting in $\mathrm{F}_{2} \mathrm{O}$, which in turn leads to $\sigma^{*}$ appearing at an energy below the lowest Rydberg orbital. This is the socalled "weak-bond effect" [2]. A high molecular strain energy induced either by perfluorination [3] or by small ring size [4] may also be effective in producing low-lying $\sigma^{*}$ MO's.

(2) In planar unsaturated systems, replacement of $\mathrm{C}-\mathrm{H}$ bonds by $\mathrm{C}-\mathrm{F}$ bonds leads to the relative stabilization of $\sigma$ MO's with respect to $\pi$ MO's. More precisely, this substitution depresses $\sigma$ MO's by $2 \mathrm{eV}$ or more while leaving $\pi$ MO energies unaffected. This so-called "perfluoro effect" upon occupied orbital energies, has been well-documented by photoelectron spectroscopy [5,

**In this paper, the highest occupied $\pi$ MO is labelled as $1 \pi$, and the lowest unoccupied $\pi$ MO as $1 \pi^{*}$. 
6]*. It also appears that a similar relative stabilization may occur in the virtual MO set, at least for outer-shell excitations, so that perfluorination of planar systems may also act to lower $\sigma^{*}$ with respect to $\pi^{*}$. Thus, in both benzene [7] and pyridine [8] anions there is a $\pi^{*}-\sigma^{*}$ crossover induced by heavy fluorination. Because bonds between fluorine and $\mathrm{N}, \mathrm{O}$, or $\mathrm{F}$ are rather weak, it is possible in these cases that the perfluoro effect (2) involves a certain amount of the weak-bond effect (1) with regard to the appearance of a low-lying $\sigma^{*}$ orbital. Note, however, that this does not apply to ethylene and the fluoroethylenes where, for example, the $\mathrm{C}-\mathrm{H}$ bond energy is $99 \mathrm{kcal} \mathrm{mol}^{-1}$ and the $\mathrm{C}-\mathrm{F}$ bond energy is $109 \mathrm{kcal} \mathrm{mol}^{-1}$ [9], or to fluorocarbons in general.

In a recent study of the effects of fluorination on the inner-shell spectrum of benzene, a rather surprising trend was noted [10]. As the benzene molecule is fluorinated, the term value of the $\mathrm{C} 1 s \rightarrow 1 \pi^{*}$ transition remains rather constant at $5-6 \mathrm{eV}$. The $\left(1 s, \chi^{*}\right)$ term value is defined as the ionization potential of the $1 s$ orbital minus the $1 s \rightarrow \chi^{*}$ excitation energy. Since the term value is equal to the $1 \pi^{*}$ excited-state ionization potential in the case of $1 s \rightarrow 1 \pi^{*}$ excitations, the constancy of the $\left(1 s, 1 \pi^{*}\right)$ term value is strongly reminiscent of the perfluoro effect as first described for outer-shell $\pi$ ionization potentials. These inner-shell spectra of the fluorobenzenes are especially interesting in that they illustrate the constancy toward fluorination of a $\pi^{*} \mathrm{MO}$ energy, compared to the previously documented constancy $[5,6]$ of $\pi$ MO energies. Moreover, these inner-shell spectra also show a $\sigma^{*}$ MO descending with increasing fluorination, again in agreement with the perfluoro effect which normally acts to stabilize $\sigma$ MO's while leaving the orbital energies of $\pi$ MO's unaffected. In as much as the explanation of the insensitivity of $\pi \mathrm{MO}$ energies to fluorination rests with a chance cancellation of inductive and conjugative (orbital overlap) effects in planar systems $[5,6,11,12]$, there is no a priori reason to expect the cancellation to be operative among $\pi^{*}$ MO's. Thus, we feel that although the inner-shell spectra of the fluorobenzenes are suggestive, there is still ample reason to question the general applicability of the perfluoro effect to inner-shell excited configurations.

It is the purpose of this paper to further investigate the applicability of the perfluoro effect to the X-ray spectra of unsaturated systems. In particular, we wish to test the constancy of the $\left(1 s, 1 \pi^{*}\right)$ term values to fluorination in both planar and nonplanar systems, and the relative stabilization of one or more $\sigma^{*}$ MO's with increasing fluorination. For this, we turn to several molecular classes that were used in the original documentation of the perfluoro effect as it applies

\footnotetext{
*Large differences of behavior upon perfluorination are observed depending upon whether all of the atoms are in the molecular plane, or one or more are not. In the context of the perfluoro effect, we refer to molecules having all atoms in a plane as "planar" and those having one or more atoms out of plane as "nonplanar", even though molecules in the latter category still might possess a reflection plane.
} 
to occupied valence levels, i.e., olefins, ketones, dienes and aromatics. Additionally, results on carboxylic acids and acetylenic compounds are included. Specifically, $\mathrm{C} 1 s$ and $\mathrm{F} 1 s$ spectra have been recorded for five fluoroethylenes, octafluorocyclopentene, formyl fluoride ( $\mathrm{HFCO}$ ), carbonyl fluoride $\left(\mathrm{F}_{2} \mathrm{CO}\right)$, trifluoroacetic acid, hexafluorobutadiene, hexafluorobutyne-2, hexafluoroacetone, and octafluoronaphthalene. $01 s$ spectra were also recorded where appropriate. These spectra were then compared with those of their perhydro analogs. Of these, the inner-shell spectra of butyne-2, naphthalene and acetic acid are reported for the first time.

In addition to considering shifts in the $\pi^{*}$ and $\sigma^{*}$ energies as related to the perfluoro effect, all spectral features of the newly obtained spectra are tentatively assigned on the basis of comparison with spectra of related molecules, expected term values and intensities for transitions terminating at Rydberg and valence orbitals [1], and the empirical correlation of $\mathrm{X}-\mathrm{Y}$ bond lengths and $\left(1 s, \sigma^{*}(\mathrm{X}-\mathrm{Y})\right)$ term values $[13,14]$.

The discussion as given above implies a uniqueness of Rydberg and $\sigma^{*} \mathrm{MO}$ 's based upon the principal quantum numbers of the AO's involved in the MO's. However, it is recognized now that this is only a zero-order description, and that there can be considerable mixing of Rydberg $R$ and valence $\sigma^{*}$ MO's depending upon their symmetries and energies $[1,15,16]$. In this work, we acknowledge the potential mixing by labelling levels capable of such mixing as $R / \sigma^{*}$ without any implication as to which component predominates. Comparison of the oscillator strengths for these features with general expectations for "pure" Rydberg transitions [1] can yield a rough measure of the extent of $R / \sigma^{*}$ mixing. The corresponding situation for $R / \pi^{*}$ mixing is not as much of a problem because Rydberg orbitals having $\pi^{*}$ symmetry ( $n d$ and $n f$ ) have very little penetration and hence will not mix strongly with $\pi^{*}$ valence MO's.

In the frozen orbital one-electron approximation, the ordering of the transitions to the virtual $\pi^{*}, \sigma^{*}$ and Rydberg MO's will be independent of the originating MO. At this level of approximation, whatever virtual-orbital ordering is determined in the inner-shell $1 s$ spectrum at several hundred $\mathrm{eV}$ energy, will apply to the interpretation of that molecule's UV spectrum in the range 3-10 $\mathrm{eV}$. Moreover, the relative locations of the $\sigma^{*}$ and $\pi^{*} \mathrm{MO}$ 's in unsaturated compounds will be evident as well in the temporary-negative-ion (TNI) spectra [17] with energies between 1 and $10 \mathrm{eV}$. Consequently, in addition to the inner-shell and UV spectra, we also consider TNI data of two types as relevant to this study; electron transmission (ET) [17] and dissociative electron attachment (DEA) spectra [18]. In the former, TNI resonances appear as abrupt changes of electron-scattering cross section as the electron energy is scanned, whereas in the latter technique, resonances are signaled by maxima in the ion-yield curves generated by scanning the impinging electron energy. The TNI resonances in ET and some of those in DEA experiments are associated with the trapping of the impinging electrons in either $\pi^{*}, \sigma^{*}$ or Rydberg 


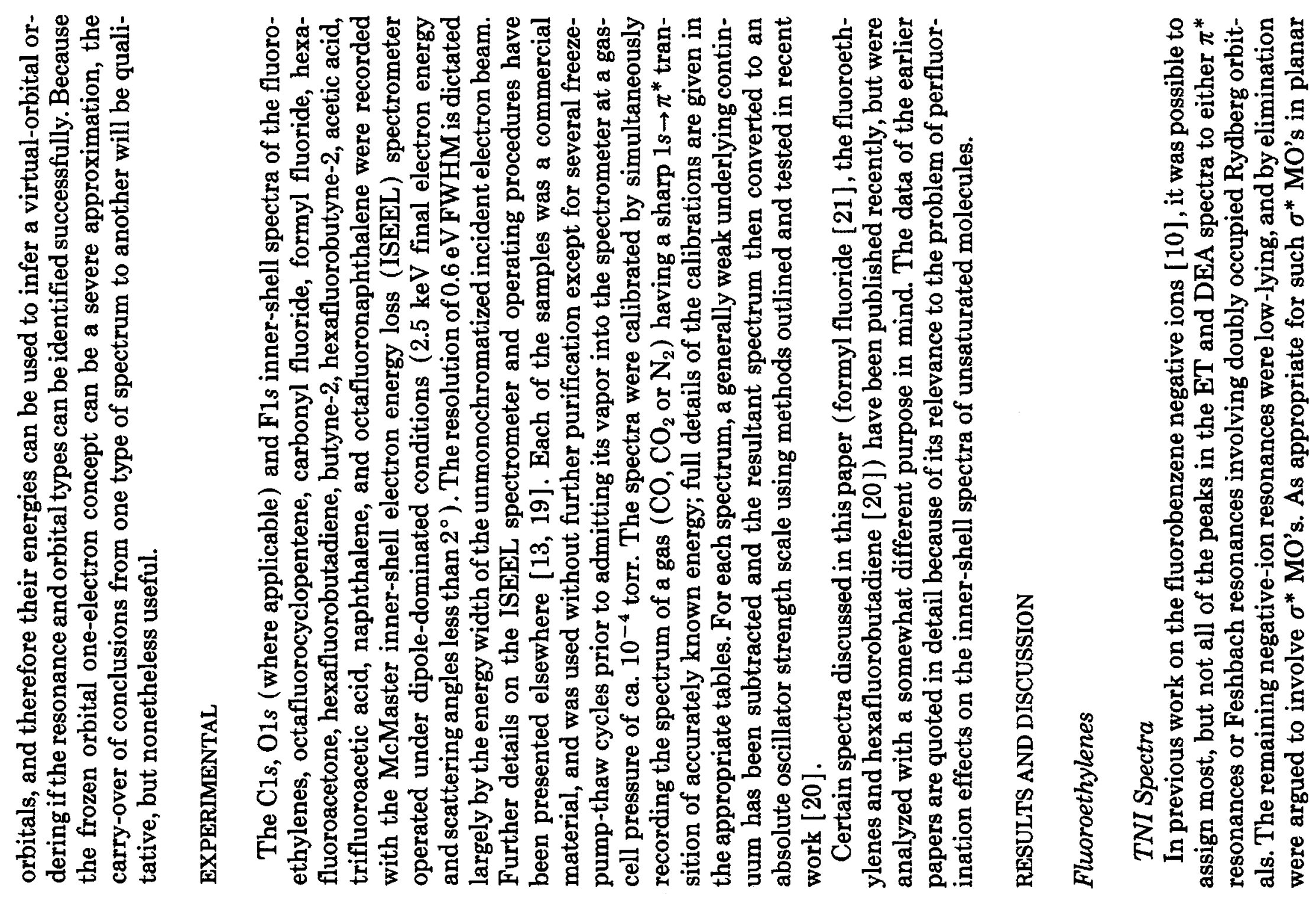




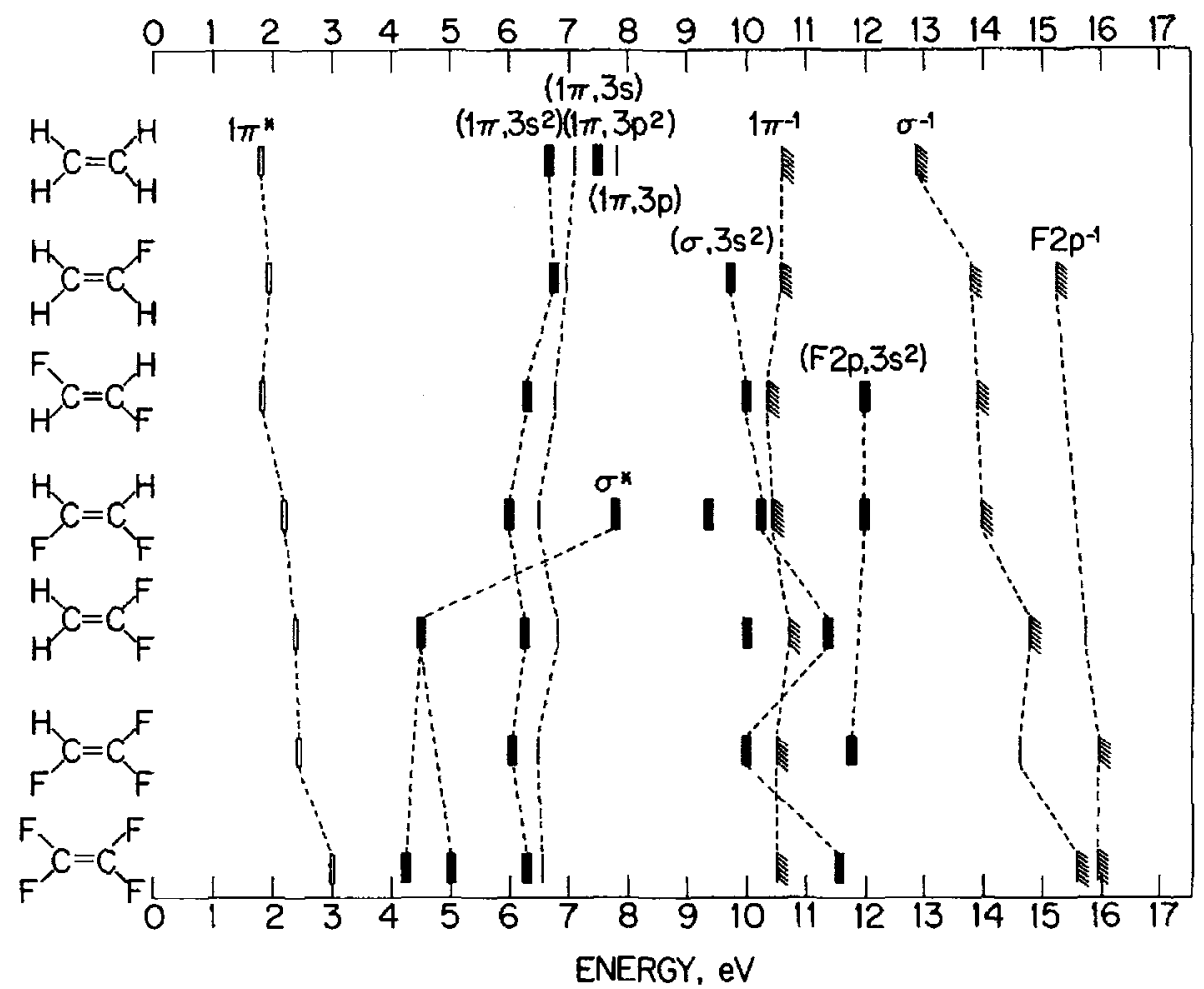

Fig. 1. Correlation of the ET resonances ( $\square$ ), DEA peaks ( $\square$ ), ionization potentials ( ) and Rydberg excited state energies (I) in ethylene and the fluoroethylenes.

systems, they were observed as low-lying only in the more heavily fluorinated species. We approach the problem of identifying $\sigma^{*}$ MO's in the fluoroethylenes in the same way.

Electron transmission resonances in the fluoroethylenes have been reported by Chiu et al. [22]; in as much as all workers in the field agree that the lowest such resonance in each of the fluoroethylenes corresponds to occupation of the $1 \pi^{*}$ MO by the incident electron, these transitions are labeled " $1 \pi$ " in Fig. 1. The lowest energy peaks found in the DEA spectra [23,24] agree closely with the ET resonances [22]. Interestingly, the trend of slightly increasing energy of the $1 \pi^{*}$ resonance with increasing degree of fluorination has been shown theoretically to correspond to the vertical excitation energies whereas the adiabatic values vary in the opposite direction [25]. The difference is associated with increasingly large geometry changes of the anion with fluorination, particularly involving displacements along the torsion coordinate leading to twisted conformations. In accord with the $n \pi-n \pi^{*}$ pairing theorem [26], the sum of the vertical $1 \pi^{-1}$ ionization potential and the vertical $1 \pi^{*}$ electron affinity for each of the fluoroethylenes $(7.5-8.3 \mathrm{eV})$ is close to the theoretical value of 8.1 $\mathrm{eV}$ predicted semiempirically.

Whereas the ET experiments yield only the lowest resonances in the fluoroethylenes, the DEA data show a number of negative-ion states beyond the 
lowest (Fig. 1). These higher resonances can be assigned once they are considered along with relevant neutral-molecule excitations. Thus, in Fig. 1, we also plot the $1 \pi^{-1}$ ionization potentials [5] along with the $1 \pi \rightarrow 3 s$ Rydberg excitation energies of the neutral fluoroethylenes [1]. The second of these curves follows the first closely, as expected for Rydberg excitations with near constant term values. The $(1 \pi, 3 s)$ line is relevant to our discussion because it is known that negative-ion Feshbach resonance configurations of the sort $\left(1 \pi, 3 s^{2}\right)$ lie about $0.5 \mathrm{eV}$ below their parent configurations $(1 \pi, 3 s)$ in both atomic and molecular systems [1]. Consequently, we have good reason to expect the $\left(1 \pi, 3 s^{2}\right)$ negative-ion energies to track the $1 \pi^{-1}$ ionization potentials and $(1 \pi, 3 s)$ excitation energies, being just $0.5 \mathrm{eV}$ below the latter. This is seen to be the case for the DEA resonances observed between 5 and $6 \mathrm{eV}$, and so identifies them as having $\left(1 \pi, 3 s^{2}\right)$ Feshbach configurations. In ethylene, a $\left(1 \pi, 3 p_{\mathbf{x}}^{2}\right)$ DEA resonance [27-29] is also seen at the expected interval below the parent $\left(1 \pi, 3 p_{\mathrm{x}}\right)$ Rydberg excitation.

The next two higher ionization potentials in the fluoroethylenes involve ejection of electrons from $\sigma$ and F2p lone pair orbitals. Repeatedly, it is the case that for each of these ionization potentials (Fig. 1) there is a corresponding $3 s^{2}$ Feshbach resonance at lower energy, the interval being very nearly that observed between $1 \pi^{-1}$ and $\left(1 \pi, 3 s^{2}\right)$. Arguing in this way, we are able to assign 17 negative-ion states in Fig. 1 to Feshbach resonances.

Once the Feshbach and $1 \pi^{*}$ resonances in ethylene and the fluoroethylenes are identified, several DEA resonances remain unassigned in the 4-5 eV. region. Since they are too low in energy to be Feshbach resonances of any sort, and since the $1 \pi^{*}$ negative ion states are preempted by the bands at $2-3 \mathrm{eV}$, there is no choice but to assign these remaining resonances to negative-ion states involving $\sigma^{*}$ MO's. The picture of virtual orbital ordering in the fluoroethylenes is thus much like that in the fluorobenzenes, with $\sigma^{*}$ MO's low-lying only in the most heavily fluorinated species. However, in the fluoroethylenes, the lowest $\sigma^{*}$ MO does not overtake $1 \pi^{*}$ as it does in the fluorobenzene series. Padden-Row et al. [25], also mention qualitatively that their calculations on the fluoroethylenes show a decreasing $\pi^{*}-\sigma^{*}$ gap as fluorination proceeds. Actually, the singly occupied $\mathrm{MO}$ in the ground state of the $\mathrm{C}_{2} \mathrm{~F}_{4}^{-}$anion in the condensed phase is found to be neither $\sigma^{*}$ nor $\pi^{*}$ but a hybrid of these appropriate to a nonplanar relaxed geometry $[25,30]$. However, this is not a significant factor in our inner-shell spectra because transitions to the short-lived core excited states are expected to be most intense at energies corresponding to the Franck-Condon maxima appropriate to planar geometries. Differences between the equilibrium geometries of the ground and core-excited states will be reflected in band widths and possibly Jahn-Teller splittings where such can be resolved [31, 32]. 


\section{C1s Inner-Shell Spectra}

The TNI spectra suggest that $\sigma^{*}$ MO's may be relatively low lying in the inner-shell spectra of the fluoroethylenes (particularly in the trifluoro and tetrafluoro compounds), possibly intruding just after the $\mathrm{C} 1 s \rightarrow 1 \pi^{*}$ transitions. The C1s and F1s inner-shell electron impact energy loss spectra of the fluoroethylenes have been reported by McLaren et al. [20], who considered both the excitation energies and their oscillator strengths; the discrete regions of the $\mathrm{C} 1 s$ fluoroethylene spectra are displayed in Fig. 2. Photoionization yields of the parent ions have also been reported in the $\mathrm{C} 1 s$ regions of these molecules [33], and show many of the same spectral features although the electron energy loss spectra appear to be of higher spectral resolution.

Looking at the C1s fluoroethylene spectra of Fig. 2, we focus on the excitations below the $\mathrm{C} 1 s$ ionization potentials (Table 1). In ethylene, there is a clear pattern of three bands $(A, B$ and $C$ ) in the $284-291 \mathrm{eV}$ region, the second of which can be resolved into two components $\left(B_{\alpha}\right.$ and $\left.B_{\beta}\right)$ in the spectrum of McLaren et al. The ethylene C1s spectrum of Tronc et al. [32], shows even finer structure due to the core splitting of the $3 p$ manifold and also to resolved vibrational activity. However, we focus on the spectra of McLaren et al., because they cover a larger number of compounds, and being of uniform resolution, they are useful in developing a broad-brush picture of the spectra when considered along with the $A B C$ classification scheme.

On the basis of term values and intensities, it is clear that band $A$ terminates at $1 \pi^{*}$, bands $B_{\alpha}$ and $B_{\beta}$ at $3 s$ and $3 p$, while band $C$ terminates at $4 p$ in ethylene. The assignment of band $A$ in olefins to $C 1 s \rightarrow 1 \pi^{*}$ is supported not only by ab initio calculations on ethylene [34], but by the out-of-plane polarization observed for the corresponding transition of st-1, 2-polybutadiene film [35], and the polarization dependence of the near-edge X-ray absorption fine structure spectrum (NEXAFS) of ethylene adsorbed onto metal surfaces [36, 37]. Although the ab initio calculations on ethylene strongly suggest that the $B_{\alpha}$ and $B_{\beta}$ bands are Rydberg excitations terminating at $3 s$ and $3 p$ [34] , they are more generally assigned here as terminating at $3 s / \sigma^{*}$ and $3 p / \sigma^{*}$, in agreement with the partial $\sigma^{*}(\mathrm{C}-\mathrm{H})$ character deduced from NEXAFS [38] and in anticipation of possible Rydberg/ $\sigma^{*}(\mathrm{C}-\mathrm{F})$ valence mixing in the fluoroethylenes. As expected from quasiatomic propensity rules, the $1 s \rightarrow 3 s$ transition of ethylene (band $B_{\alpha}$ ) is weaker than the $1 s \rightarrow 3 p$ transition (band $B_{\beta}$ ).

Going to the other end of the fluoroethylene series, an apparently similar pattern of excitations to $A, B$ and $C$ again is seen in $\mathrm{C}_{2} \mathrm{~F}_{4}$ (Fig. 2a), this time between 289 and $295 \mathrm{eV}$. Because the $\mathrm{C} 1 s$ orbital in this case originates within a $\mathrm{CF}_{2}$ group, the bands are labelled $A^{\prime \prime}, B^{\prime \prime}$ and $C^{\prime \prime}$; in the case of excitation from a CHF group, the terminology is $A^{\prime}, B^{\prime}$ and $C^{\prime}$. The $B^{\prime \prime}$ band of $C_{2} F_{4}$ is broad and rather asymmetric, and could be resolved further into $B_{\alpha}{ }^{\prime \prime}$ and $B_{\beta}{ }^{\prime \prime}$ components through graphical decomposition into Gaussian sub-bands, Fig. 2a. The spectrum of cis-CHFCHF shows a similar $A^{\prime} B^{\prime} C^{\prime}$ pattern with a clear 


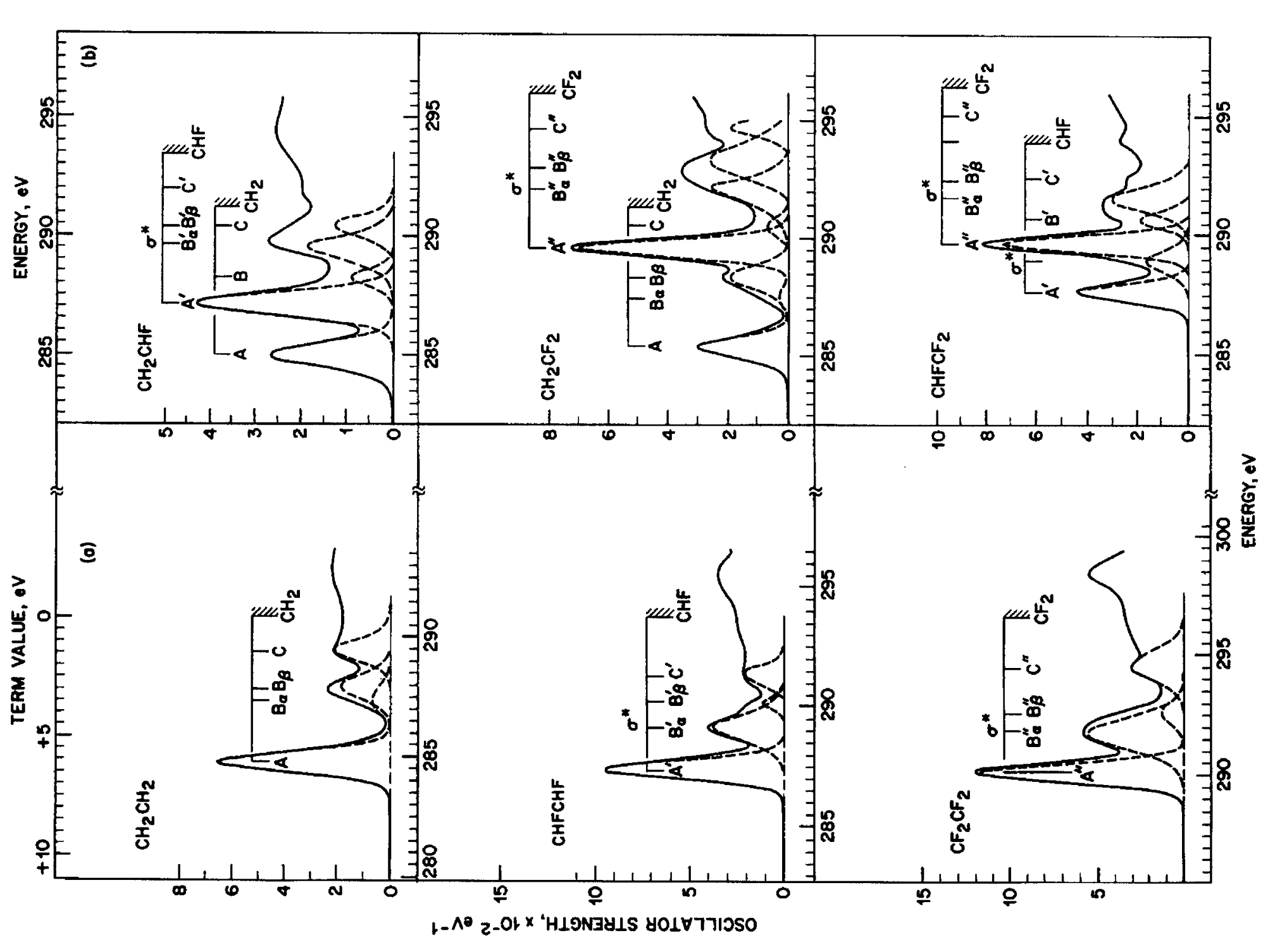

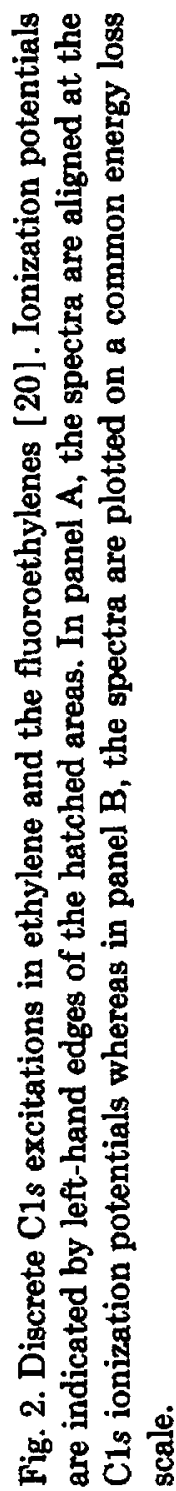




\section{TABLE 1}

Energies $(\Delta E, \mathrm{eV})$ and term values $(\mathrm{TV}, \mathrm{eV})^{\mathrm{a}}$ in the $\mathrm{C} 1 s$ spectra of ethylene and its fluorinated derivatives $\mathrm{s}^{\mathrm{b}, \mathrm{c}}$

\begin{tabular}{|c|c|c|c|c|c|c|c|c|c|c|c|c|c|c|c|}
\hline & \multicolumn{12}{|l|}{ Band } & \multirow{2}{*}{$\begin{array}{l}\mathrm{IP} \\
\left(\mathrm{CH}_{2}\right)\end{array}$} & \multirow[t]{2}{*}{ (CHF) } & \multirow[t]{2}{*}{$\left(\mathrm{CF}_{2}\right)^{\prime \prime}$} \\
\hline & $A$ & $A^{\prime}$ & $A^{\prime \prime}$ & $B_{\alpha}$ & $B_{\alpha}^{\prime}$ & $B_{\alpha}{ }^{\prime \prime}$ & $B_{\beta}$ & $B_{\beta}^{\prime}$ & $B_{\beta}^{\prime \prime}$ & $C$ & $C^{\prime}$ & $C^{\prime \prime}$ & & & \\
\hline \multicolumn{16}{|c|}{$\mathrm{H}_{2} \mathrm{CCH}_{2}$} \\
\hline$\Delta E$ & 284.65 & & & 287.4 & & & 287.8 & & & 289.3 & & & 290.88 & & \\
\hline TV & 6.23 & & & 3.5 & & & 3.1 & & & 1.6 & & & & & \\
\hline \multicolumn{16}{|c|}{$\mathrm{H}_{2} \mathrm{CCHF}$} \\
\hline$\Delta E$ & 284.95 & 287.10 & & & 289.8 & & & & & & 291.9 & & 291.10 & 293.48 & \\
\hline TV & 6.15 & 6.38 & & & 3.7 & & & & & & 1.6 & & & & \\
\hline \multicolumn{16}{|c|}{ FHCCHF } \\
\hline$\Delta E$ & & 287.25 & & & 289.1 & & 290.1 & & & & 291.2 & & & $293.7^{\mathfrak{c}}$ & \\
\hline TV & & 6.4 & & & 4.6 & & 3.6 & & & & 2.5 & & & & \\
\hline \multicolumn{16}{|c|}{$\mathrm{H}_{2} \mathrm{CCF}_{2}$} \\
\hline$\Delta E$ & 285.41 & & 289.58 & 287.5 & & 292.2 & 288.3 & & 292.9 & & & 294.8 & 291.33 & & 296.10 \\
\hline TV & 5.92 & & 6.52 & 3.8 & & 3.9 & 3.0 & & 3.2 & & & 1.3 & & & \\
\hline \multicolumn{16}{|c|}{ FHCCF $_{2}$} \\
\hline$\Delta E$ & & 287.63 & 289.64 & & & 291.4 & & & 292.4 & & & 294.1 & & 293.87 & 296.25 \\
\hline TV & & 6.24 & 6.61 & & & 4.1 & & & 3.1 & & & 2.1 & & & \\
\hline \multicolumn{16}{|c|}{$\mathrm{F}_{2} \mathrm{CCF}_{2}$} \\
\hline$\Delta E$ & & & 290.13 & & & 291.9 & & & 292.5 & & & 294.5 & & & 296.54 \\
\hline TV & & & 6.41 & & & 4.6 & & & 4.0 & & & 2.0 & & & \\
\hline
\end{tabular}

aTerm values of bands assigned as unprimed, primed or double primed are calculated with respect to the ionization potentials of $\mathrm{CH}_{2}$, $\mathrm{CHF}_{\text {or }} \mathrm{CF}_{2}$ groups, respectively. ${ }^{b} \mathrm{This}$ Table lists only the first four bands preceding each C1s ionization potential. (See [20] for a complete list of spectral features and detailed assignments.) ${ }^{\circ}$ Ionization potentials taken from ref. 39 , except for cis-CHFCHF which was estimated from those of $\mathrm{CH}_{2} \mathrm{CHF}^{-}$ and $\mathrm{CHFCF}_{2}$. 
subdivision of the $B^{\prime}$ band into $B_{\alpha}{ }^{\prime}$ and $B_{\beta}{ }^{\prime}$ components. Spectra of the other three intermediate fluoroethylenes (Fig. 2b) are complicated by having two dissimilar $\mathrm{C} 1 s$ ionization potentials [39]. Nonetheless, using term values we are able to recognize the $A B C$ pattern associated with the second ionization limit in these compounds, and some if not all of this pattern associated with the first. The spectral decomposition in the case of $\mathrm{CH}_{2} \mathrm{CHF}$ (Fig. 2b) is consistent with its $\mathrm{C} 1 s$ spectrum taken at higher resolution [40].

Viewing the term values in aggregate (Table 1), those of the $A, A^{\prime}$ and $A^{\prime \prime}$ bands $\left(\mathrm{C} 1 s \rightarrow 1 \pi^{*}\right)$ are largely constant in the 5.9-6.6 eV range. Considering that the inductive effect of perfluorinating ethylene is to shift the C1s ionization potential from 290.88 to $296.54 \mathrm{eV}$, it is rather remarkable that the (C1s, $1 \pi^{*}$ ) ionization potential, i.e., term value is constant in the fluoroethylene series to within $\pm 0.35 \mathrm{eV}$ (Table 1 ). This shows in a dramatic way that the perfluoro effect is operating for the $\left(\mathrm{C} 1 s, 1 \pi^{*}\right)$ configurations of the fluoroethylenes, just as it is for the corresponding $\left(\mathrm{C} 1 s, 1 \pi^{*}\right)$ and $\left(\mathrm{C} 1 s, 2 \pi^{*}\right)$ configurations in the fluorobenzenes [10].

Knowing that the perfluoro effect is working for the inner-shell ( $\left.\mathrm{C} 1 s, 1 \pi^{*}\right)$ excited states of the fluoroethylenes, it is reasonable to expect that a $\left(\mathrm{C} 1 s, \sigma^{*}\right)$ excited state might drop in energy as fluorination proceeds. Our interest now is in finding excitations at or below the inner-shell ionization potentials which differ from the $A B C$ features of ethylene, for such features are most likely to be the excitations terminating at $\sigma^{*}$. According to the negative-ion study discussed above, there is ample reason to expect such $\sigma^{*}$ MO's in the heavily fluorinated ethylenes to lie just above the $1 \pi^{*} \mathrm{MO}$. Yet another possibility is that the heavily fluorinated ethylenes will not show a distinct transition to $\sigma^{*}$, but that one of the $B_{\alpha}, B_{\beta}$ or $C$ Rydberg bands of ethylene will assume more valence character with fluorination, becoming a $1 s \rightarrow \sigma^{*}$ transition in the more heavily fluorinated derivatives. Just such a change of character has been predicted by Schwarz et al. [15], who argue that upon fluorinating $\mathrm{CH}_{4}$ to form $\mathrm{CH}_{3} \mathrm{~F}$, the $3 s$ Rydberg $\mathrm{MO}$ of the former becomes a totally symmetric $\sigma^{*}$ valence $\mathrm{MO}$ in the latter. The $\mathrm{C} 1 s$ spectrum of $\mathrm{CH}_{3} \mathrm{~F}$ [41] shows a broad band with a high-energy shoulder in the $3 s / \sigma^{*}(\mathrm{C}-\mathrm{F})$ region, indicating the existence of two transitions, probably with mixed Rydberg/valence character in each [15]. Were this tendency toward increased valence character to persist or increase up to full fluorination and were the ethylene case parallel to that of methane, then one might take the $B$ bands of the more highly fluorinated fluoroethylenes as involving the sought-for $\sigma^{*}$ MO's. Of course, all degrees of $3 s / \sigma^{*}$ mixed character are possible.

Though $\mathrm{C}_{2} \mathrm{H}_{4}$ and $\mathrm{C}_{2} \mathrm{~F}_{4}$ both show the $A B_{\alpha} B_{\beta} C$ pattern of bands, it is important to note that the $B_{\alpha}$ band $(1 s \rightarrow 3 s)$ of ethylene is much weaker than the $B_{\beta}$ band $(1 s \rightarrow 3 p)$, as expected from the quasiatomic selection rules, whereas the $B_{\alpha}$ " band in tetrafluoroethylene is much stronger than the $B_{\beta}{ }^{\prime \prime}$ band. In addition, the $B$ group is shifted to considerably larger term value in $\mathrm{C}_{2} \mathrm{~F}_{4}$. We take 
the intensity and term value anomalies as evidence that the $B_{\alpha}{ }^{\prime \prime}$ band of $\mathrm{C}_{2} \mathrm{~F}_{4}$, nominally $1 s \rightarrow 3 s$, is either overlaid by or is transformed into one having strong $1 s \rightarrow 2 p \sigma^{*}(\mathrm{C}-\mathrm{F})$ character. The apparent change of atomic orbital symmetry is acceptable if one considers the localization of the $\mathrm{C} 1 s$ hole and the inherent inequivalence of the two $\mathrm{C}$ atoms in the excited state. The $B_{\alpha}{ }^{\prime}$ band at 289.0 $\mathrm{eV}$ in cis-HFCCHF also has enhanced intensity and increased term value relative to $B_{\alpha}$ of $\mathrm{C}_{2} \mathrm{H}_{4}$, signalling a C1s $\rightarrow 2 p \sigma^{*}(\mathrm{C}-\mathrm{F})$ transition. Going on to the species with distinguishable $\mathrm{C} 1 s$ ionization potentials (Fig. $2 \mathrm{~b}$ ) in $\mathrm{F}_{2} \mathrm{CCHF}$ there is a band which does not fit the $A B C$ pattern; it just follows behind the corresponding $1 s \rightarrow 1 \pi^{*}$ transition and is assigned as $1 s \rightarrow \sigma^{*}$. The situation in $\mathrm{H}_{2} \mathrm{CCF}_{2}$ resembles that in $\mathrm{C}_{2} \mathrm{~F}_{4}$, i.e., the transition energies fit the $A B_{\alpha} B_{\beta} C$ pattern nicely, however the $B_{\alpha}{ }^{\prime \prime}$ band again is much more intense than one expects for $1 s \rightarrow 3 s$, and so is assigned as $1 s \rightarrow 3 s / \sigma^{*}$. Features identified by curve fitting of the $\mathrm{CH}_{2} \mathrm{CHF}$ and $\mathrm{CF}_{2} \mathrm{CHF}$ spectra [20] have been tentatively assigned as $\mathrm{C} 1 s(\mathrm{CHF}) \rightarrow \sigma^{*}(\mathrm{C}-\mathrm{F})$ following consideration of their term values and increased intensities.

The presence of inner-shell transitions to $\sigma^{*}$ in the fluoroethylenes at or near those to $3 s$ closely resembles the situation in the fluoromethanes [1, 42]. In both series of compounds, fluorination is accompanied by increasing $\mathrm{C} 1 s \rightarrow \sigma^{*}$ (C-F) intensity and increasing distinguishability of these from the C1s-toRydberg transitions. As expected from earlier work [1], the (C1s, $\left.3 s / \sigma^{*}(\mathrm{C}-\mathrm{F})\right)$ inner-shell Rydberg term values increase somewhat with fluorination, rising from $3.5 \mathrm{eV}$ in $\mathrm{C}_{2} \mathrm{H}_{4}$ to $4.6 \mathrm{eV}$ in $\mathrm{C}_{2} \mathrm{~F}_{4}$. These inner-shell term values are exhalted over those observed for the same molecules in the $(1 \pi, 3 s)$ outer-shell configurations [1].

Support for the reality of low lying inner-shell transitions to $\sigma^{*} \mathrm{MO}$ 's in the fluoroethylenes comes from their vacuum UV spectra [1, 43]. After assigning the $\pi \rightarrow 3 s$ and $\pi \rightarrow \pi^{*}$ transitions on the basis of their term values, intensities and response to external perturbation, yet another transition is observed between the transitions to $3 s$ and $\pi^{*}$ in cis- and trans-difluoro-, trifluoro- and tetrafluoroethylene. Unlike the Rydberg excitations in the fluoroethylenes, the transition lying between those to $3 s$ and $\pi^{*}$ persists in the condensed phase as is characteristic of a valence excitation, and has been given a $\pi \rightarrow \sigma^{*}$ assignment [1]. The term values for these $\pi \rightarrow \sigma^{*}$ valence excitations are between 2.9 and $3.6 \mathrm{eV}$, to be compared with $3.5-4.6 \mathrm{eV}$ for the $\mathrm{C} 1 s \rightarrow \sigma^{*}$ excitations deduced from Fig. 2. Due to inner-shell exhaltation [1], the term values of inner-shell transitions are usually ca. $1 \mathrm{eV}$ or so larger than the outer-shell counterparts terminating at the same MO's.

\section{F1s Inner-Shell Spectra}

In the F1s spectra of the fluoroethylenes [20], the features assigned as $\mathrm{F} 1 s \rightarrow 1 \pi^{*}$ excitations have reduced term values of $4.0-5.4 \mathrm{eV}$ as compared with the 5.9-6.6 eV range in the $\mathrm{C} 1 s$ spectra. Similar decreases of the $\left(\mathrm{F} 1 s, 1 \pi^{*}\right)$ 
term values with respect to $\left(\mathrm{C} 1 s, 1 \pi^{*}\right)$ have also been observed in the fluorobenzenes [10], the ranges being 3.5-5.0 eV and 4.9-6.2 eV, respectively. The second band of the F1s spectra has a range of term values $(2.2-2.8 \mathrm{eV})$ appropriate to $\mathrm{F} 1 s \rightarrow 3 p / \sigma^{*}$ excitation, and indeed these features were assigned to $\mathrm{F} 1 s \rightarrow \sigma^{*}(\mathrm{C}-\mathrm{F})$ in [20]. As with the fluorobenzenes, resolved features assignable to $\mathrm{F} 1 s \rightarrow 3 s$ transitions do not appear in the spectra of the fluoroethylenes.

\section{Fluoroalkyl Ethylenes}

In planar molecules in which the perfluoro effect is known to work on valence-shell ionization potentials, it is also observed that perfluorination of their methyl derivatives results in out-of-plane $F$ atoms which destroy the $\pi-\sigma$ symmetry distinction through mixing of the $\pi$ and $\sigma$ manifolds. Consequently, in such molecules the " $\pi$ " MO's shift downward on fluorination by amounts that rival those for " $\sigma$ " MO's. Given this valence-shell behavior, it is of interest to compare the inner-shell $\left(1 s, 1 \pi^{*}\right)$ term values in methylated olefins and their perfluorinated derivatives.

On going from butene-2 to its perfluorinated derivative, the $\pi \mathrm{MO}$ binding energy increases from 9.11 to $11.55 \mathrm{eV}$ [5]. This $1 \pi$ energy difference of 2.44 $\mathrm{eV}$ induced by perfluorination of nonplanar butene-2 is far larger than the 0.1 $\mathrm{eV}$ shift of the $1 \pi$ level induced by perfluorination of the planar molecule ethylene. As for the effect of perfluorination on the $1 \pi^{*} \mathrm{MO}$ energy in these nonplanar molecules, the $\left(\mathrm{C} 1 s, 1 \pi^{*}\right)$ term value increases from 5.7 to $6.8 \mathrm{eV}$ [13]. Though this is a large shift in the expected direction, and is far larger than that observed on going from ethylene to tetrafluoroethylene, it is not as large as that observed for the $1 \pi$ valence MO. Still, it does show that the perfluorination shift of inner-shell $\left(\mathrm{C} 1 s, 1 \pi^{*}\right)$ term values is strongly dependent upon whether any of the fluorine atoms lie out of the $\pi$-orbital nodal plane, just as for the valence-shell binding energies.

Comparison of the inner-shell spectra of cyclopentene [4] and of octafluorocyclopentene allows another probe of the effect of geometry on the unoccupied $\pi^{*}$ and $\sigma^{*}$ orbital energies. The C1s and F1s spectra of octafluorocyclopentene appear in Fig. 3 together with the $\mathrm{C} 1 s$ spectrum of cyclopentene, while the energies, term values and proposed assignments of the octafluorocyclopentene spectral features are given in Table 2 . The lowest feature in the $\mathrm{C} 1 s$ spectrum is attributed to a $\mathrm{C} 1 s$ (CF) $\rightarrow 1 \pi^{*}$ promotion; its term value of 7.5 $\mathrm{eV}$ is $2.1 \mathrm{eV}$ larger than that of the $\mathrm{C} 1 s \rightarrow 1 \pi^{*}$ transition of cyclopentene [4]. As is the case with valence-shell ionization potentials, it appears that the (C1s, $1 \pi^{*}$ ) inner-shell term values are considerably enhanced upon perfluorination in systems where the $\sigma(\mathrm{C}-\mathrm{F})$ bonds and antibonds are oriented out-of-plane and so mix with $1 \pi^{*}$.

The equality of the $7.5 \mathrm{eV}$ term value of peak 2 of octafluorocyclopentene with respect to the $\mathrm{C} 1 s\left(\mathrm{CF}_{2}\right)$ ionization potential and that of peak 1 with re- 


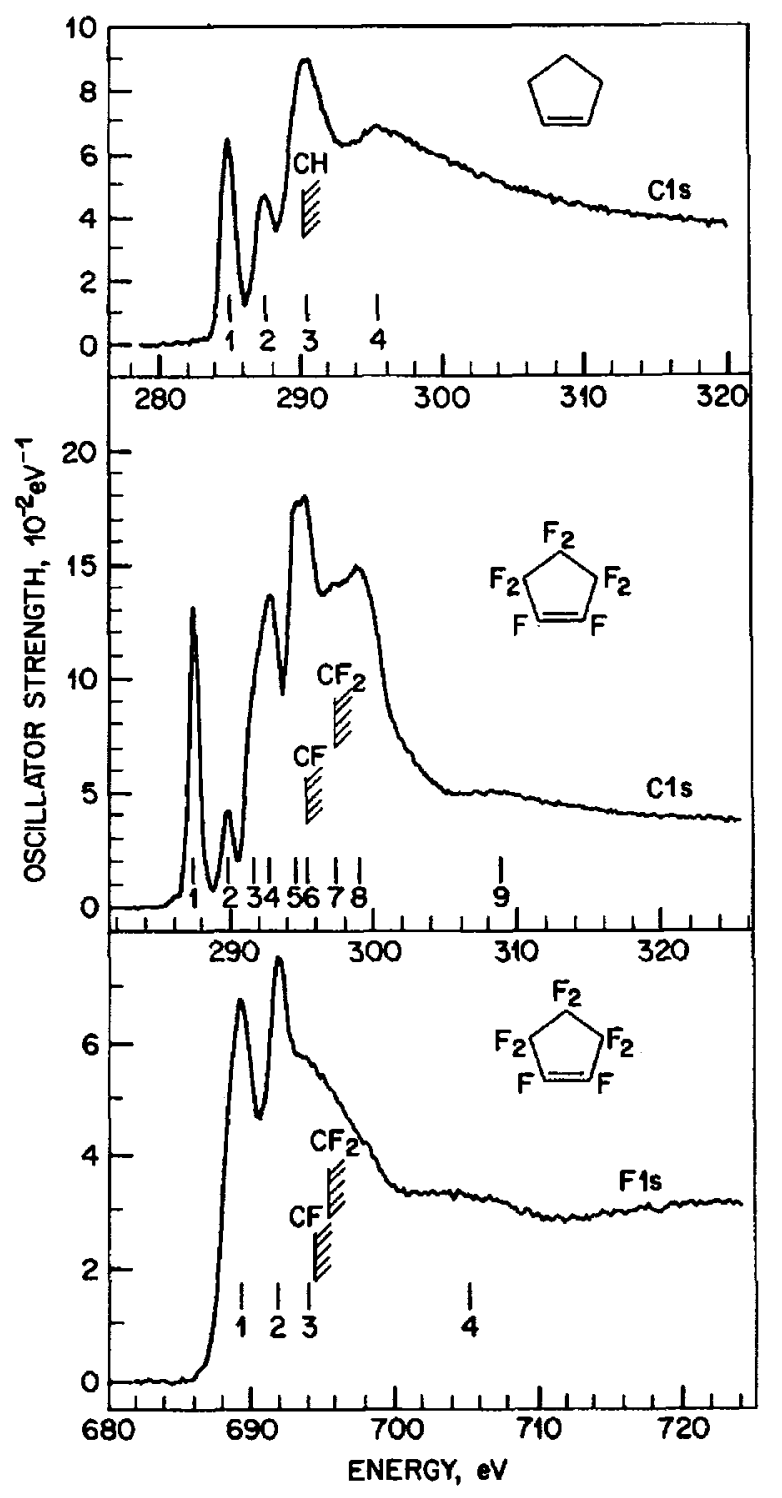

Fig. 3. $\mathrm{C} 1 s$ oscillator strength distributions derived from the ISEEL spectra of cyclopentene (upper panel) and octafluorocyclopentene (middle panel) and the F1s distribution for octafluorocyclopentene (lower panel). All energy loss spectra were recorded with $2.5 \mathrm{keV}$ final electron energy, $2^{\circ}$ average scattering angle and 0.6 FWHM resolution.

spect to $\mathrm{C} 1 s(\mathrm{CF})$ at first argues for a $\mathrm{C} 1 s\left(\mathrm{CF}_{2}\right) \rightarrow 1 \pi^{*}$ assignment for peak 2 . However, we reject this assignment on the basis that the $\left\langle\mathrm{C} 1 s\left(\mathrm{CF}_{2}\right)|\mu| 1 \pi^{*}\right\rangle$ transition matrix element will be extremely small because of poor orbital overlap, and that the term value for such a charge-transfer transition should be significantly reduced from that of the on-site $\mathrm{C} 1 s(\mathrm{CF}) \rightarrow 1 \pi^{*}$ transition (vide infra). If the $\mathrm{C} 1 s\left(\mathrm{CF}_{2}\right) \rightarrow 1 \pi^{*}$ transition has detectable intensity, we believe it will contribute in the region of shoulder $3(5.6 \mathrm{eV}$ term value). Peak 2 of the $\mathrm{C} 1 s$ spectrum of octafluorocyclopentene is assigned to $\mathrm{C} 1 s(\mathrm{CF}) \rightarrow 3 s / \sigma^{*}(\mathrm{C}-$ F) with a $5.1 \mathrm{eV}$ term value, parallel to the $B_{\alpha}^{\prime}$ feature in the fluoroethylene spectra. 
TABLE 2

Energies $(\Delta E, \mathrm{eV})$, term values $(\mathrm{TV}, \mathrm{eV})$ and proposed assignments for 1 s excitation in octafluorocyclopentene

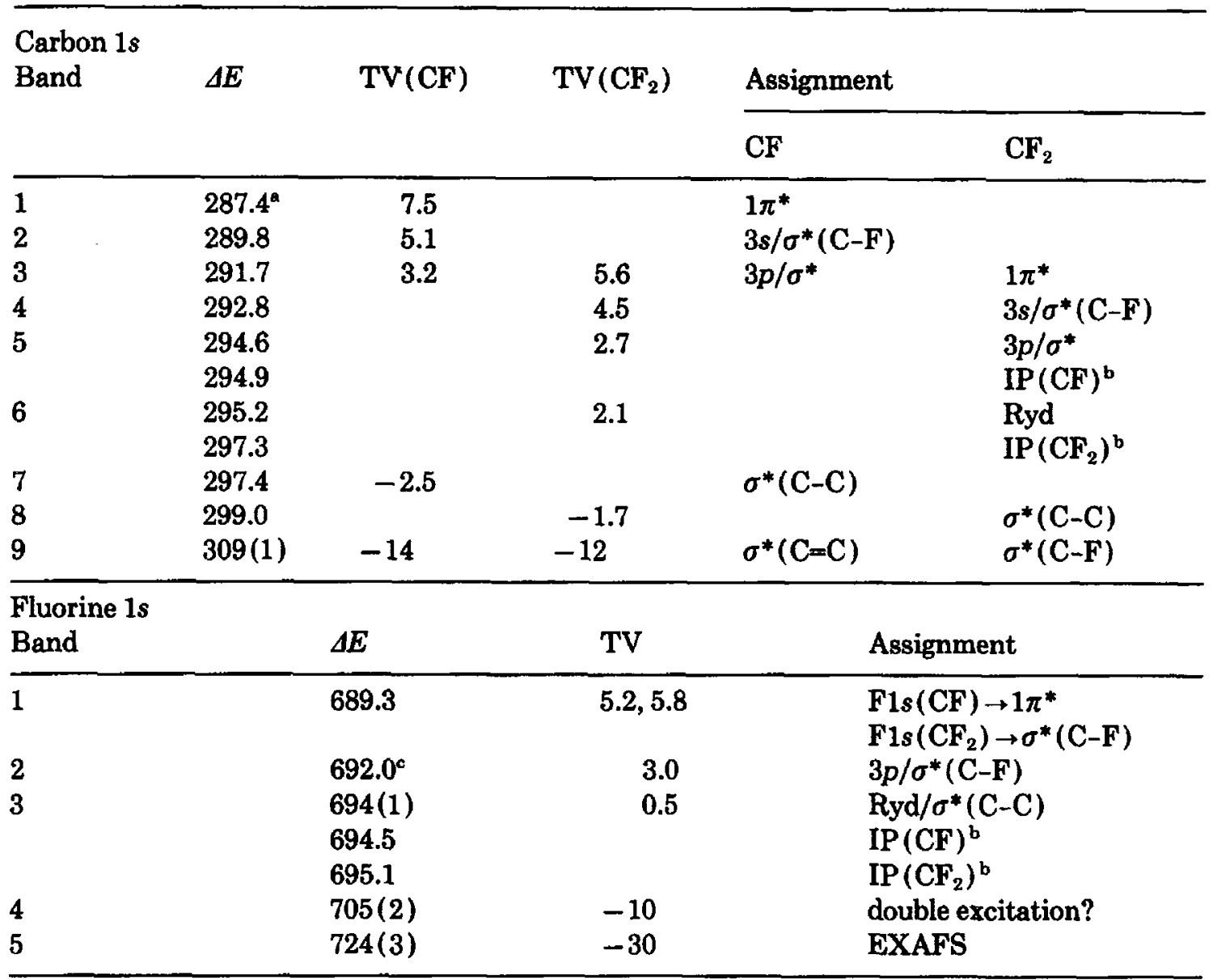

aCalibrated as $3.32(5) \mathrm{eV}$ below the $\mathrm{C} 1 s \rightarrow 1 \pi^{*}$ band of $\mathrm{CO}_{2}(290.74 \mathrm{eV}) .{ }^{\text {b }}$ Taken from X-ray photoelectron spectra [39]. "Calibrated as 156.6(2) eV above the $\mathrm{O} 1 s \rightarrow 1 \pi^{*}$ band in $\mathrm{CO}_{2}(535.4$ $\mathrm{eV})$.

Note too that band 2 of octafluorocyclopentene is similar to the second band in the C1s spectrum of perfluorobutene-2 [13], which previously had been assigned as $\mathrm{C} 1 s\left(\mathrm{CF}_{3}\right) \rightarrow 1 \pi^{*}$ with a term value of $9.0 \mathrm{eV}$. We believe this assignment is incorrect both because of the expected small overlap of the $\mathrm{C} 1 s\left(\mathrm{CF}_{3}\right)$ and $1 \pi^{*}$ orbitals and because of its unrealistically large term value (that for $\mathrm{C} 1 s(\mathrm{CF}) \rightarrow 1 \pi^{*}$ is only $6.8 \mathrm{eV}$ ). Instead, the assignment of this feature to $\mathrm{C} 1 s(\mathrm{CF}) \rightarrow 3 s / \sigma^{*}(\mathrm{C}-\mathrm{F})$ with a $4.2 \mathrm{eV}$ term value, an alternative discussed in reference [13], appears more reasonable based on comparison with the spectra of this study. The higher energy losses in the C1s spectrum of octafluorocyclopentene are attributed to excitations to $3 s / \sigma^{*}(\mathrm{C}-\mathrm{F}), 3 p / \sigma^{*}$ and $\sigma^{*}(\mathrm{C}-\mathrm{C})$ orbitals from each of the two distinguishable $\mathrm{C} 1 \mathrm{~s}$ levels. The continuum peak at $309 \mathrm{eV}$ terminates at the $\sigma^{*}(\mathrm{C}=\mathrm{C}) \mathrm{MO}$. 
The first peak in the F1s spectrum of octafluorocyclopentene is attributed to the overlap of $\mathrm{F} 1 s(\mathrm{CF}) \rightarrow 1 \pi^{*}$ and $\mathrm{F} 1 s\left(\mathrm{CF}_{2}\right) \rightarrow \sigma^{*}(\mathrm{C}-\mathrm{F})$ transitions, with the latter probably contributing the majority of the intensity. As with the other $\left(\mathrm{F} 1 s, 1 \pi^{*}\right)$ term values discussed in this work, that of octafluorocyclopentene $(5.2 \mathrm{eV})$ is significantly smaller than that of the corresponding $\mathrm{C} 1 s \rightarrow 1 \pi^{*}$ transition $(7.5 \mathrm{eV})$. We attribute the diminution of the $\left(\mathrm{F} 1 s, 1 \pi^{*}\right)$ term value to the charge-transfer character of this transition as compared with that of $\mathrm{C} 1 s \rightarrow 1 \pi^{*}$. The second relatively sharp feature in the $\mathrm{F} 1 s$ spectrum of octafluorocyclopentene is assigned as $\mathrm{F} 1 s \rightarrow 3 p / \sigma^{*}(\mathrm{C}-\mathrm{F})$. Several strong $\mathrm{F} 1 s \rightarrow \sigma^{*}(\mathrm{C}-\mathrm{F})$ features are consistently observed in the spectra of perfluoroalkanes [3], indicating a significant splitting among the $\sigma^{*}(\mathrm{C}-\mathrm{F})$ orbitals. This contrasts with the relatively small splitting of the $\sigma^{*}(\mathrm{C}-\mathrm{C})$ manifold of linear alkanes as evidenced by the observation of a single dominant $\mathrm{C} 1 s \rightarrow \sigma^{*}(\mathrm{C}-\mathrm{C})$ shape resonance in ethane which broadens relatively slowly with increasing chain length [44]. Feature 3 at $694 \mathrm{eV}$ in octafluorocyclopentene is attributed to the overlap of $\mathrm{F} 1 s \rightarrow \sigma^{*}(\mathrm{C}-\mathrm{C})$ and higher Rydberg transitions. The features in the F1s continuum could be two-electron excitations and/or EXAFS interferences.

\section{Aldehydes and Ketones}

The compounds $\mathrm{H}_{2} \mathrm{CO}, \mathrm{HFCO}$ and $\mathrm{F}_{2} \mathrm{CO}$ are of special interest in this study since they are planar and isoelectronic with $\mathrm{H}_{2} \mathrm{CCH}_{2}, \mathrm{HFCCH}_{2}$ and $\mathrm{F}_{2} \mathrm{CCH}_{2}$. Oscillator strength distributions derived from the inner-shell electron-energy loss spectra of $\mathrm{F}_{2} \mathrm{CO}$ are presented in Fig. 4, Table 3, while the $\mathrm{C} 1 s$ and $\mathrm{O} 1 \mathrm{~s}$ spectra of all three species are presented in Fig. 5 and Table 3. The $\mathrm{H}_{2} \mathrm{CO}$ results are derived from the published spectrum of Hitchcock and Brion [45], and the $\mathrm{HFCO}$ spectrum was recently published in a comparison with $\mathrm{HCONH}_{2}$ and $\mathrm{HCOOH}[21]$.

In the olefin series, fluorination of $\mathrm{H}_{2} \mathrm{CCH}_{2}$ to form $\mathrm{F}_{2} \mathrm{CCH}_{2}$ raises the (C1s, $1 \pi^{*}$ ) term value from 6.23 to $6.52 \mathrm{eV}$, an upward shift of only $0.29 \mathrm{eV}$. Similar fluorination of $\mathrm{H}_{2} \mathrm{CO}$ to form $\mathrm{F}_{2} \mathrm{CO}$ raises the $\left(\mathrm{C} 1 s, 1 \pi^{*}\right)$ term value from 8.46 to $8.75 \mathrm{eV}$, an upward shift of only $0.29 \mathrm{eV}$. Thus, the substitution of $\pi$-bonding to carbon with $\pi$-bonding to an electronegative heteroatom such as oxygen raises the $\left(\mathrm{C} 1 s, 1 \pi^{*}\right)$ term value considerably. However, as with ethylene, this term value in formaldehyde is quite insensitive to subsequent fluorination. Looking at the other end of the $\mathrm{C}=\mathrm{O}$ double bond, the $01 s$ spectra show transitions to $1 \pi^{*}$ with term values which are similar in $\mathrm{H}_{2} \mathrm{CO}(8.64 \mathrm{eV})$ and $\mathrm{F}_{2} \mathrm{CO}$ $(8.1 \mathrm{eV})$, and which closely match those of $\mathrm{C} 1 s \rightarrow 1 \pi^{*}$ in the same molecules, Table 3. These observations are again in excellent agreement with expectations with regard to the perfluoro effect.

Yet another constancy of the fluoroethylene data [20] is the relatively depressed value of the $\left(\mathrm{F} 1 s, 1 \pi^{*}\right)$ term value compared to $\left(\mathrm{C} 1 s, 1 \pi^{*}\right)$. Thus, looking at $\mathrm{F}_{2} \mathrm{CCH}_{2}$, the $\left(\mathrm{F} 1 s, 1 \pi^{*}\right)$ term value is $2.3 \mathrm{eV}$ smaller than the (C1s, 

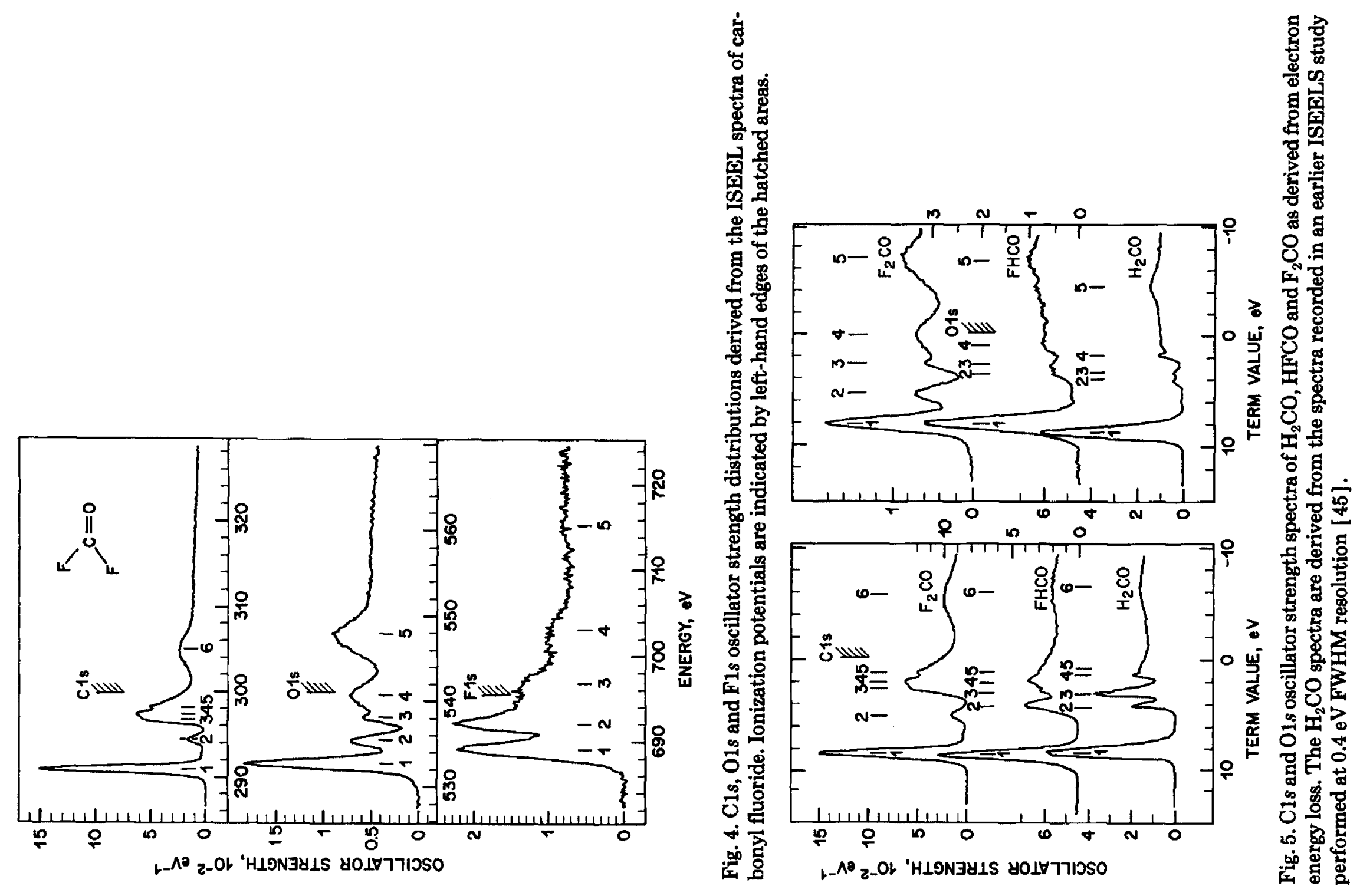
TABLE 3

Energies $(\Delta E, \mathrm{eV})$, term values $(\mathrm{TV}, \mathrm{eV})$ and proposed assignments of inner-shell excitation in formaldehyde,", formyl fluoride ${ }^{\mathrm{b}}$ and carbonyl fluoride.

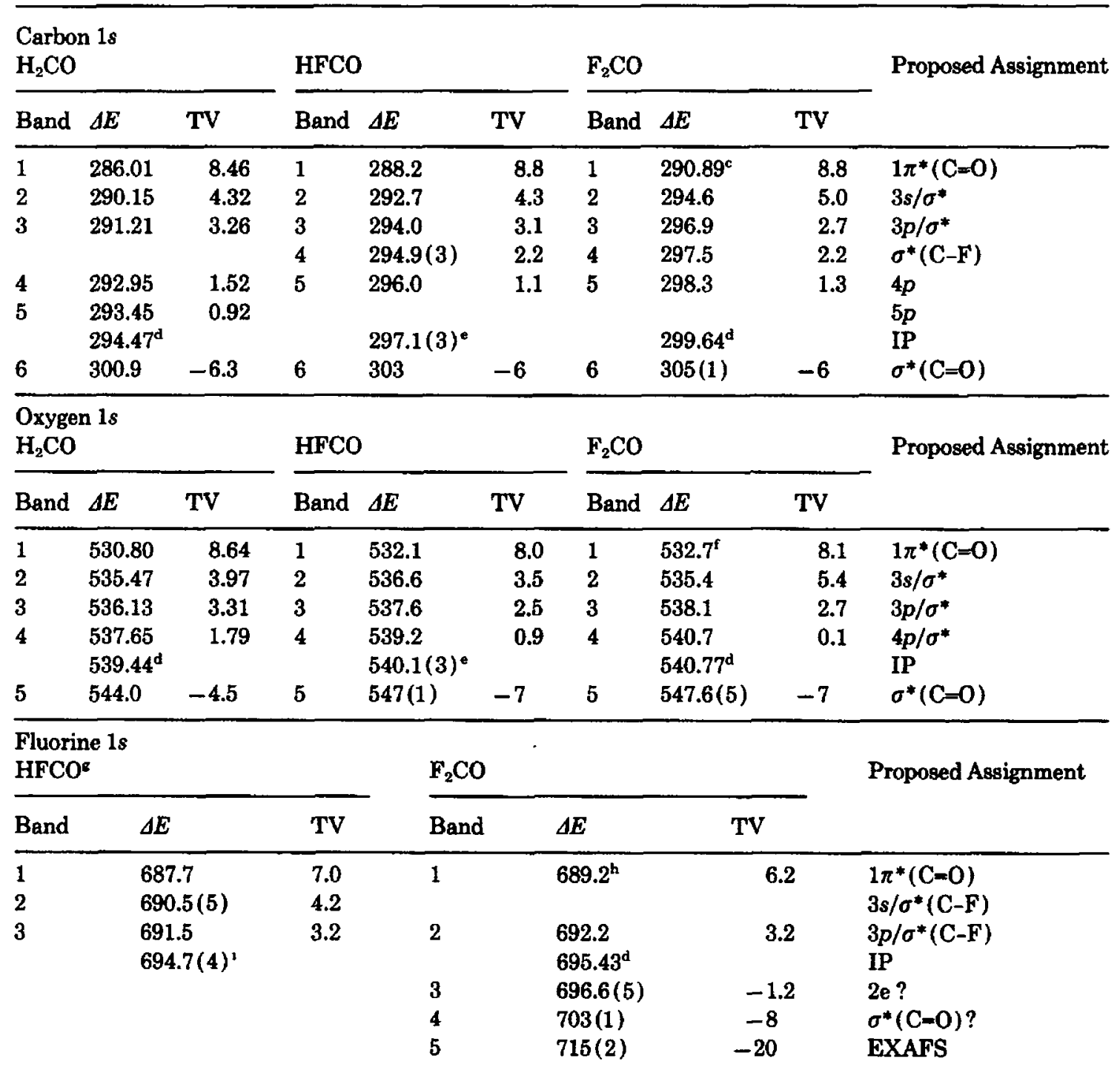

-Numerical data from [45]. Note the numbering schemes do not correspond since only the principal features are listed in this paper. 'From [21]. 'Calibrated as $3.49(5) \mathrm{eV}$ above the $\mathrm{C} 1 \mathrm{~s} \rightarrow 1 \pi^{*}$ transition of CO. ${ }^{d}$ From [39]. 'Estimated as the average of the ionization potentials of $\mathrm{H}_{2} \mathrm{CO}$ and $\mathrm{F}_{2} \mathrm{CO}$ [20]. ${ }^{\mathrm{f}} \mathrm{Calibrated}$ as 1.91(5) $\mathrm{eV}$ above the $01 s \rightarrow 1 \pi^{*}$ transition of $\mathrm{O}_{2}(530.8 \mathrm{eV}) .{ }^{\mathrm{s} S e e}[21]$ for the plot of the F1s spectrum of HFCO. h'Calibrated as $156.5 \mathrm{eV}$ above the $\mathrm{Ol} \rightarrow 1 \pi^{*}$ transition of $\mathrm{CF}_{3} \mathrm{COCF}_{3}$. 'Estimated after consideration of the Fls ionization potentials of $\mathrm{F}_{2} \mathrm{CO}, \mathrm{CH}_{2} \mathrm{~F}_{2}$ and $\mathrm{CH}_{3} \mathrm{~F}[20]$.

$1 \pi^{*}$ ) term value of $6.52 \mathrm{eV}$. In an exactly parallel manner, the term value for ( $\left.\mathrm{F} 1 s, 1 \pi^{*}\right)$ of $\mathrm{F}_{2} \mathrm{CO}$ is $2.5 \mathrm{eV}$ smaller than that for $\left(\mathrm{C} 1 s, 1 \pi^{*}\right)$ of $\mathrm{H}_{2} \mathrm{CO}$. The inner-shell spectra of the intermediate system $\mathrm{FHCO}$ show term values slightly larger than those reported here for $\mathrm{F}_{2} \mathrm{CO}$, however these have been calculated using estimated ionization potentials [21]. 


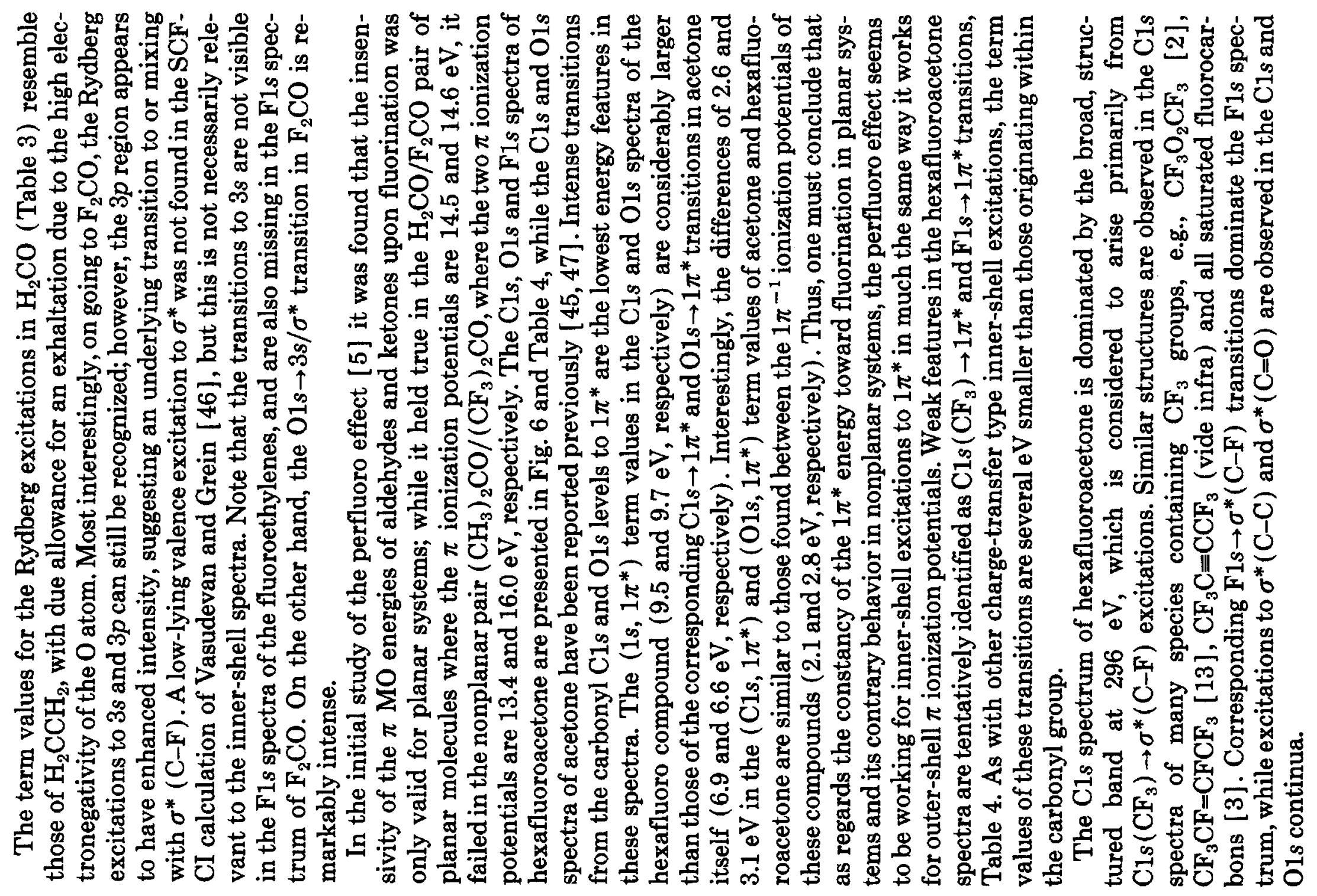




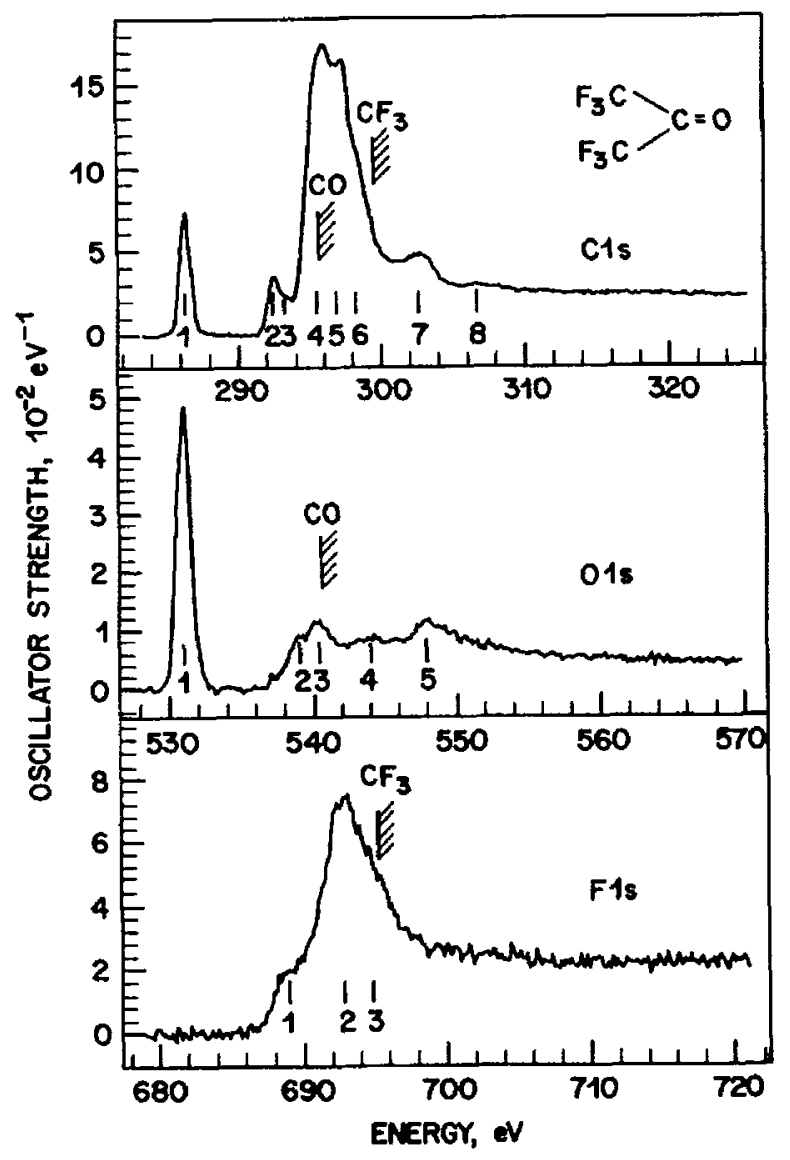

Fig. 6. $\mathrm{C} 1 s, \mathrm{O} 1 s$ and $\mathrm{F} 1 s$ oscillator strength distributions derived from ISEEL spectra of hexafluoroacetone. Ionization potentials are indicated by left-hand edges of hatched areas.

\section{Butadiene}

It is known that perfluorination of butadiene does not result in the $\pi$-orbital behavior expected for the perfluoro effect, the reason being that perfluorination strongly twists the molecule out of its planar form, leading to valence orbital shifts more like those that typify fluorination of nonplanar unsaturates [48]. The effect of nonplanarity is at work as well in the inner-shell spectra of these dienes; comparison of the spectra of $\mathrm{C}_{4} \mathrm{H}_{6}$ and $\mathrm{C}_{4} \mathrm{~F}_{6}$ [20], shows the $\left(\mathrm{C} 1 s, 1 \pi^{*}\right)$ term value of $\mathrm{C}_{4} \mathrm{H}_{6}(5.8 \mathrm{eV})$ increases to $7.0 \mathrm{eV}$ in $\mathrm{C}_{4} \mathrm{~F}_{6}$. Such a high value of the $\left(\mathrm{C} 1 s, 1 \pi^{*}\right)$ term value in a perfluoro unsaturate is characteristic of a nonplanar geometry, as illustrated above by the comparison of the inner-shell spectra of butene-2, cyclopentene and acetone with their perfluorinated derivatives.

Butyne-2

Being linear rather than planar, the acetylene/difluoroacetylene pair is a special case in regard the perfluoro effect. Nonetheless, as expected, perfluor- 


\section{TABLE 4}

Absolute energies $(\Delta E, \mathrm{eV})$, term values ( $\mathrm{TV}, \mathrm{eV}$ ) and proposed assignments for hexafluoroacetone

\begin{tabular}{|c|c|c|c|c|c|}
\hline \multicolumn{6}{|c|}{ Carbon $1 s$} \\
\hline \multirow[t]{2}{*}{ Band } & \multirow[t]{2}{*}{$\Delta E$} & \multicolumn{2}{|l|}{ TV } & \multicolumn{2}{|c|}{ Proposed Assignment } \\
\hline & & $\mathrm{Cls}(\mathrm{CO})$ & $\mathrm{Cls}\left(\mathrm{CF}_{3}\right)$ & $\mathrm{C} 1 s(\mathrm{CO})$ & $\mathrm{C} 1 s\left(\mathrm{CF}_{3}\right)$ \\
\hline 1 & $286.2^{\mathrm{a}}$ & 9.5 & & $1 \pi^{*}(\mathrm{C}=0)$ & \\
\hline 2 & 292.4 & 3.4 & 7.0 & $3 s$ & $1 \pi^{*}(\mathrm{C}=0)$ \\
\hline \multirow[t]{2}{*}{3} & $293.5(10)$ & 2.2 & & & \\
\hline & 295.75 & & & $\mathrm{IP}^{\mathrm{h}}$ & \\
\hline 4 & 295.9 & -0.2 & 3.5 & $\sigma^{*}(\mathrm{C}-\mathrm{C})$ & $3 s / \sigma^{*}(\mathrm{C}-\mathrm{F})$ \\
\hline 5 & 297.2 & & 2.2 & & $3 p / \sigma^{*}(\mathrm{C}-\mathrm{F})$ \\
\hline \multirow[t]{2}{*}{6} & $298.5(3)$ & & 0.9 & & $\sigma^{*}(\mathrm{C}-\mathrm{C})$ \\
\hline & 299.41 & & & & $\mathrm{IP}^{\mathrm{b}}$ \\
\hline 7 & $302.6(3)$ & -6.8 & & $\sigma^{*}(\mathrm{C}=0)$ & \\
\hline 8 & $306.4(5)$ & & -7.0 & & $\sigma^{*}(\mathrm{C}=0)$ or $2 \mathrm{e}^{-}$ \\
\hline \multicolumn{6}{|c|}{ Oxygen $1 s$} \\
\hline Band & $\Delta E$ & TV & \multicolumn{3}{|c|}{ Proposed Assignment } \\
\hline 1 & $531.0^{c}$ & 9.7 & \\
\hline 2 & 539.0 & 1.6 & \multicolumn{3}{|c|}{$3 p / \sigma^{*}(\mathrm{C}-\mathrm{F})$} \\
\hline 3 & 540.4 & 0.3 & \multicolumn{3}{|c|}{$\sigma^{*}(\mathrm{C}-\mathrm{C}) / \sigma^{*}(\mathrm{C}-\mathrm{F})$} \\
\hline & 540.65 & & \multicolumn{3}{|c|}{$\mathrm{IP}^{\mathrm{b}}$} \\
\hline 4 & $543.8(4)$ & -3.2 & \multicolumn{3}{|l|}{$2 e^{-}$} \\
\hline 5 & $548.2(6)$ & -7.6 & \multicolumn{3}{|l|}{$\sigma^{*}(\mathrm{C}=0)$} \\
\hline \multicolumn{6}{|c|}{ Fluorine $1 s$} \\
\hline Band & $\Delta E$ & TV & \multicolumn{3}{|c|}{ Proposed Assignment } \\
\hline 1 & 688.7 & 6.3 & \multicolumn{3}{|l|}{$1 \pi^{*}(\mathrm{C}=0)$} \\
\hline 2 & $692.8^{d}$ & 2.2 & \multicolumn{3}{|l|}{$\sigma^{*}(\mathrm{C}-\mathrm{F})$} \\
\hline \multirow[t]{2}{*}{3} & $695(1)$ & 0 & \multicolumn{3}{|l|}{$\sigma^{*}(\mathrm{C}-\mathrm{C})$} \\
\hline & 695.08 & & \multicolumn{3}{|l|}{$\mathrm{IP}^{\mathrm{b}}$} \\
\hline
\end{tabular}

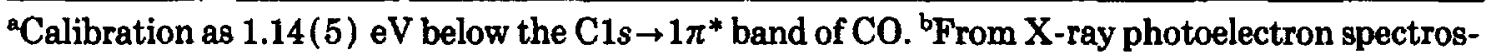
copy [39]. ${ }^{\circ}$ Calibrated as $4.44(5) \mathrm{eV}$ below the $\mathrm{O} 1 s \rightarrow 1 \pi^{*}$ band of $\mathrm{CO}_{2} \cdot{ }^{\mathrm{d}} \mathrm{Calibrated}$ as $161.9(2)$ $\mathrm{eV}$ above band 1 of the $01 s$ spectrum.

ination of acetylene does not shift the $1 \pi$ valence $M O$ energy [49]; it is expected that this would be the case as well for excitations to $1 \pi^{*}$ from C1s in these compounds. It follows from the planar-molecule behavior of acetylene that the $1 \pi$ and $1 \pi^{*}$ levels of the nonlinear (nonplanar) methyl derivative butyne- 2 will be appreciably stabilized when perfluorinated. The $\mathrm{C} 1 s$ spectrum of butyne-2 and the C1s and F1s spectra of its hexafluoro derivative are presented in Fig. 7, while the energies, term values and proposed assignments are listed in Table 5. The first three transitions in butyne-2 terminate at 


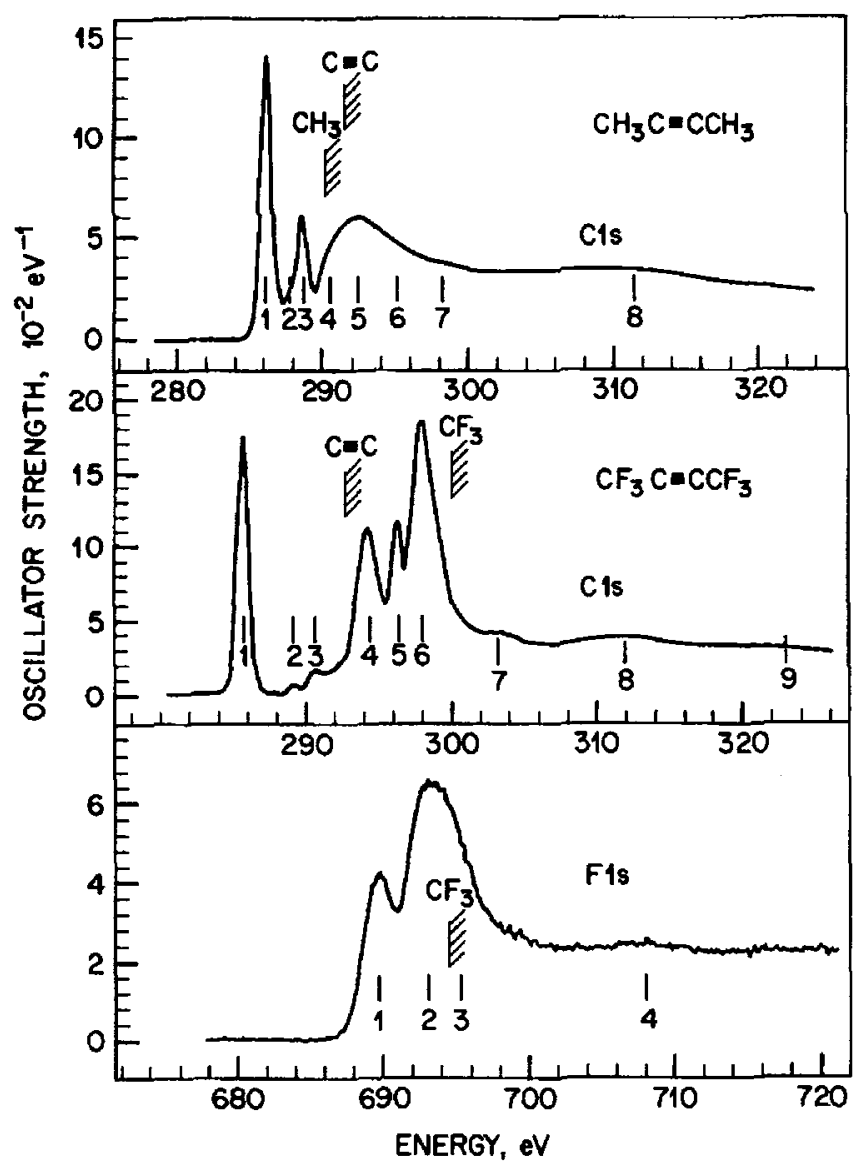

Fig. 7. Oscillator strength distributions derived from the ISEEL spectra of butyne-2 (C1s) and of hexafluorobutyne-2 ( $\mathrm{C} 1 s$ and $\mathrm{F} 1 s)$. Ionization potentials are indicated by left-hand edges of hatched areas.

$\left(\mathrm{C} 1 s(\mathrm{C} \equiv \mathrm{C}), 1 \pi^{*}\right),\left(\mathrm{C} 1 s\left(\mathrm{CH}_{3}\right), 3 p\right)$ and $(\mathrm{C} 1 s(\mathrm{C} \equiv \mathrm{C}), 3 p)$ configurations, respectively. The $\left(\mathrm{C} 1 s, 1 \pi^{*}\right)$ term value of $5.18 \mathrm{eV}$ is $0.5 \mathrm{eV}$ smaller than the corresponding $\left(\mathrm{C} 1 s, 1 \pi^{*}\right)$ term values of cis- and trans-butene-2 [13], as is characteristic of the relative term values of alkenes and alkynes in general [20].

In hexafluorobutyne-2, the $\mathrm{C} 1 s$ ionization potentials are separated by $7.2 \mathrm{eV}$ [38], leading to a clear separation of the $\mathrm{C} 1 s$ features originating at $1 s(\mathrm{C} \equiv \mathrm{C})$ (bands $1-3,8)$ and $1 s\left(\mathrm{CF}_{3}\right)$ (bands $\left.4-7\right)$. One finds the (C1s $\left.(\mathrm{C} \equiv \mathrm{C}), 1 \pi^{*}\right)$ term value of butyne-2 to have increased to $7.0 \mathrm{eV}$ due to the perfluorination, while in butene-2, perfluorination raises the $\left(\mathrm{C} 1 s, 1 \pi^{*}\right)$ term value from 5.8 to $7.0 \mathrm{eV}$. It is seen in butyne-2 that once again the fluorination of methyl groups resulting in out-of-plane $\mathrm{F}$ atoms increases the $\left(\mathrm{C} 1 s, 1 \pi^{*}\right)$ term value by ca. $2 \mathrm{eV}$. Comparison of the photoelectron spectra of butyne- 2 and hexafluorobutyne-2 shows as well that perfluorination increases the $1 \pi$ valence orbital binding energy by $2.76 \mathrm{eV}$ [50].

Feature 4 in the C1s spectrum of hexafluorobutyne-2 (Fig. 7) is attributed 


\section{TABLE 5}

Energies $(\Delta E, \mathrm{eV})$, term values $(\mathrm{TV}, \mathrm{eV})$ and assignments for the inner-shell spectra of butyne2 and hexafluorobutyne-2

\begin{tabular}{|c|c|c|c|c|c|}
\hline \multicolumn{6}{|c|}{ Butyne-2 (C1s) } \\
\hline \multirow[t]{2}{*}{ Band } & \multirow[t]{2}{*}{$\Delta E$} & \multicolumn{2}{|l|}{ TV } & \multicolumn{2}{|l|}{ Assignment } \\
\hline & & $\left(\mathrm{CX}_{3}\right)$ & $(\mathrm{C} \equiv \mathrm{C})$ & $\left(\mathrm{CX}_{3}\right)$ & $(\mathrm{C}=\mathrm{C})$ \\
\hline 1 & $286.12^{\mathrm{a}}$ & 3.91 & 5.18 & $3 s$ & $1 \pi^{*}$ \\
\hline 2 & 287.7 & 2.3 & & $3 p$ & \\
\hline 3 & 288.5 & & 2.8 & & $3 p$ \\
\hline \multirow[t]{3}{*}{4} & $290.5(3)$ & & 0.8 & & Ryd \\
\hline & $290.03^{b}$ & & & IP & \\
\hline & $291.30^{\mathrm{b}}$ & & & & IP \\
\hline 5 & 292.3 & -2.3 & & $\sigma^{*}(\mathrm{C}-\mathrm{C})$ & \\
\hline 6 & 295.3 & & -4.0 & & $\sigma^{*}(\mathrm{C}-\mathrm{C})$ \\
\hline 7 & $298.0(5)$ & & -6.7 & & $2 e^{-}$ \\
\hline 8 & $311.4(5)$ & & -20.1 & & $\sigma^{*}(\mathrm{C}=\mathrm{C})$ \\
\hline \multicolumn{6}{|c|}{ Hexafluorobutyne-2 (C1s) } \\
\hline 1 & $285.7^{c}$ & & 7.0 & & $1 \pi^{*}$ \\
\hline 2 & 289.1 & & 3.6 & & $3 s$ \\
\hline \multirow[t]{2}{*}{3} & 290.6 & & 2.1 & & $3 p$ \\
\hline & $292.7^{b}$ & & & & IP \\
\hline 4 & 294.3 & 5.6 & -1.6 & $1 \pi^{*} / \sigma^{*}(\mathrm{C}-\mathrm{F})$ & $\sigma^{*}(\mathrm{C}-\mathrm{C})$ \\
\hline 5 & 296.3 & 3.6 & & $3 s / \sigma^{*}(\mathrm{C}-\mathrm{F})$ & \\
\hline \multirow[t]{2}{*}{6} & 298.0 & 1.9 & -5.3 & $3 p / \sigma^{*}(\mathrm{C}-\mathrm{F})$ & $\sigma^{*}(\mathrm{C}-\mathrm{C})$ \\
\hline & $299.9^{b}$ & & & IP & \\
\hline 7 & 303.3 & -3.4 & & $\sigma^{*}(\mathrm{C}-\mathrm{C})$ & \\
\hline 8 & $312(1)$ & & -19 & & $\sigma^{*}(\mathrm{C} \equiv \mathrm{C})$ \\
\hline 9 & $323(1)$ & & & & EXAFS \\
\hline
\end{tabular}

Hexafluorobutyne-2 (F1s)

\begin{tabular}{llrl}
\hline 1 & $689.8^{\mathrm{d}}$ & 4.6 & $3 s / \sigma^{*}(\mathrm{C}-\mathrm{F}), 1 \pi^{*}$ \\
2 & 693.3 & 1.2 & $3 p / \sigma^{*}(\mathrm{C}-\mathrm{F})$ \\
3 & 695.1 & 0.6 & $\sigma^{*}(\mathrm{C}-\mathrm{C})$ \\
& $694.5^{\mathrm{b}}$ & & IP \\
4 & 708 & -14 & EXAFS \\
\hline
\end{tabular}

${ }^{a}$ Calibrated to be $1.28(3) \mathrm{eV}$ below the $\mathrm{C} 1 s \rightarrow 1 \pi^{*}$ band of $\mathrm{CO}$ at $287.40 \mathrm{eV}$. ${ }^{\mathrm{b}} \mathrm{From}$ [39]. ${ }^{\mathrm{C}}$ Calibrated as $1.72 \mathrm{eV}$ below the $\mathrm{C} 1 s \rightarrow 1 \pi^{*}$ band of $\mathrm{CO}$ at $287.40 \mathrm{eV}$. ${ }^{\mathrm{d}}$ Calibrated as $155.6(2) \mathrm{eV}$ above the $01 s \rightarrow 1 \pi^{*}$ transition of $\mathrm{CO}$ at $534.2 \mathrm{eV}$.

to the overlap of $\mathrm{C} 1 s(\mathrm{C} \equiv \mathrm{C}) \rightarrow \sigma^{*}(\mathrm{C}-\mathrm{C})$ and $\mathrm{C} 1 s\left(\mathrm{CF}_{3}\right) \rightarrow 1 \pi^{*}$ excitations. Promotions to a low-lying $\sigma^{*}(\mathrm{C}-\mathrm{F})$ MO may also contribute here. The $\mathrm{C} 1 s\left(\mathrm{CF}_{3}\right) \rightarrow 1 \pi^{*}$ term value is $5.6 \mathrm{eV}$, while that from the first $\mathrm{F} 1 s$ feature is only $4.6 \mathrm{eV}$. In both cases, these term values are much smaller than the $\mathrm{C} 1 s(\mathrm{C} \equiv \mathrm{C}) \rightarrow 1 \pi^{*}$ term value $(7.0 \mathrm{eV})$, suggesting that the larger the spatial 
separation of the $1 s$ and $1 \pi^{*} \mathrm{MO}$ 's, the higher the excitation energy with respect to the appropriate $1 s$ ionization potential. That is to say, charge transfer in the upper state decreases the term value. This generalization is supported by the data on the fluoroethylenes [20] which show the $\left(\mathrm{C} 1 s, 1 \pi^{*}\right)$ term values to be significantly larger than the corresponding ( $\left.\mathrm{F} 1 s, 1 \pi^{*}\right)$ term values. In contrast, the $\mathrm{F} 1 s \rightarrow \sigma^{*}(\mathrm{O}-\mathrm{F})$ transitions of $\mathrm{F}_{2} \mathrm{O}$ do not involve any significant charge transfer, and so have term values equal to those originating at $01 s$ [2].

In both butyne- 2 and hexafluorobutyne-2, the $\mathrm{C} 1 s \rightarrow \sigma^{*}(\mathrm{C}-\mathrm{C})$ transitions are assigned as features with term values of -2 to $-5 \mathrm{eV}$, rather higher than the term values which are close to $0 \mathrm{eV}$ for the corresponding transitions in other hydrocarbons. This is consistent with the bond length/term value correlation and the reduced C-C bond distance of only $1.48 \AA$ in these butynes. In addition, our assignment of the very high-lying $\mathrm{C} 1 s$ continuum features as $\mathrm{C} 1 s \rightarrow \sigma^{*}(\mathrm{C} \equiv \mathrm{C}$ ) (band 8 ) with term values of ca. $-20 \mathrm{eV}$ relative to the $\mathrm{C} 1 s(\mathrm{C} \equiv \mathrm{C})$ ionization potential is supported by the bond length/term value correlation and the $\mathrm{C} \equiv \mathrm{C}$ bond distance of $1.20 \AA$.

\section{Aromatics}

\section{Benzene}

The inner-shell spectra of benzene and its fluoro derivatives have been compared in reference [10], along with data on negative-ion resonances. This study showed that with progressive fluorination of benzene, a $\sigma^{*}$ MO drops between $1 \pi^{*}$ and $2 \pi^{*}$, and in the hexafluorobenzene anion it actually falls below $1 \pi^{*}$. In accord with the perfluoro effect, the $\left(\mathrm{C} 1 s, 1 \pi^{*}\right)$ term values are relatively insensitive to fluorination, increasing from $5.1 \mathrm{eV}$ in benzene to only $6.2 \mathrm{eV}$ in hexafluorobenzene. The excitation from $\mathrm{F} 1 s$ is not as regular, with ( $\left.\mathrm{F} 1 s, 1 \pi^{*}\right)$ term values ranging from $3.5 \mathrm{eV}$ (benzene) to $5.0 \mathrm{eV}$ (hexafluorobenzene). Once again, these are smaller than the corresponding $\left(\mathrm{C} 1 s, 1 \pi^{*}\right)$ term values due to the charge transfer nature of the $\mathrm{F} 1 s \rightarrow 1 \pi^{*}$ transitions. It was concluded that with respect to $\mathrm{C} 1 s$ excitation, the $\left(1 s, 1 \pi^{*}\right)$ and $\left(1 s, \sigma^{*}\right)$ term values on fluorination of benzene behave as expected for the perfluoro effect in a planar system [10].

\section{Naphthalene}

The C1s spectra of naphthalene and the $\mathrm{C} 1 s$ and F1s spectra of octafluoronaphthalene are presented in Figs. 8 and 9, while the energies, term values relative to estimated ionization potentials, oscillator strengths and proposed assignments are presented in Table 6 . The $\mathrm{C} 1 s$ spectrum of naphthalene exhibits four relatively sharp features and a shoulder below the estimated $\mathrm{C} 1 s$ ionization potential. These are attributed to the five possible $\mathrm{C} 1 s \rightarrow n \pi^{*} \operatorname{tran}$ sitions. A good match (Fig. 9) is observed with the term values and relative 


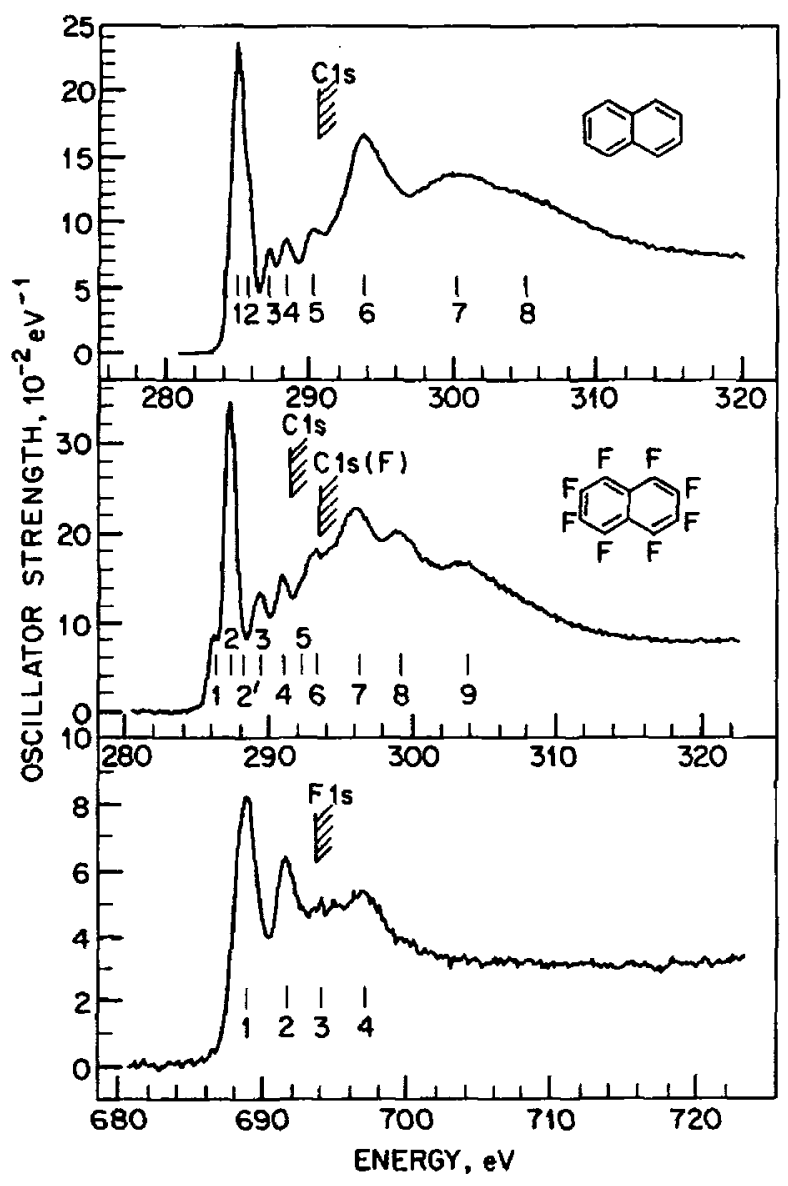

Fig. 8. Comparison of the $\mathrm{C} 1 s$ oscillator strength spectrum of naphthalene with the C1s and F1s oscillator strength spectra of octafluoronaphthalene. Ionization potentials are as estimated in the footnote to Table 6 .

intensities predicted by a HAM/ 3 calculation $[51,52]$ and with the relative orbital energies predicted by a Hückel calculation in which $\beta=-3.20 \mathrm{eV}$ is assumed.

The C1s NEXAFS spectrum of a multilayer naphthalene film on an $\mathrm{Ag}$ (111) surface recently has been reported [53]. The five $\mathrm{C} 1 s \rightarrow n \pi^{*}$ transitions which we observe in the free molecule appear in the low-resolution NEXAFS as two bands. The polarization dependence of these transitions in the film is consistent with out-of-plane polarized $\mathrm{C} 1 s \rightarrow n \pi^{*}$ excitations in naphthalene molecules having their molecular planes parallel to the $\mathrm{Ag}$ (111) surface, although the second NEXAFS band has some residual intensity at normal incidence, suggesting an underlying in-plane $\mathrm{C} 1 s \rightarrow \sigma^{*}(\mathrm{C}-\mathrm{H})$ component.

The inner-shell spectrum of octafluoronaphthalene is complicated by the existence of two $\mathrm{C} 1 s$ environments with resolvable ionization potentials. Though excitations from the eight $\mathrm{C}-\mathrm{F}$ carbons will dominate the spectrum, some of those originating at the two bridgehead $\mathrm{C}$ atoms nonetheless can be resolved. If the second peak in the spectrum of the octafluoro derivative is 


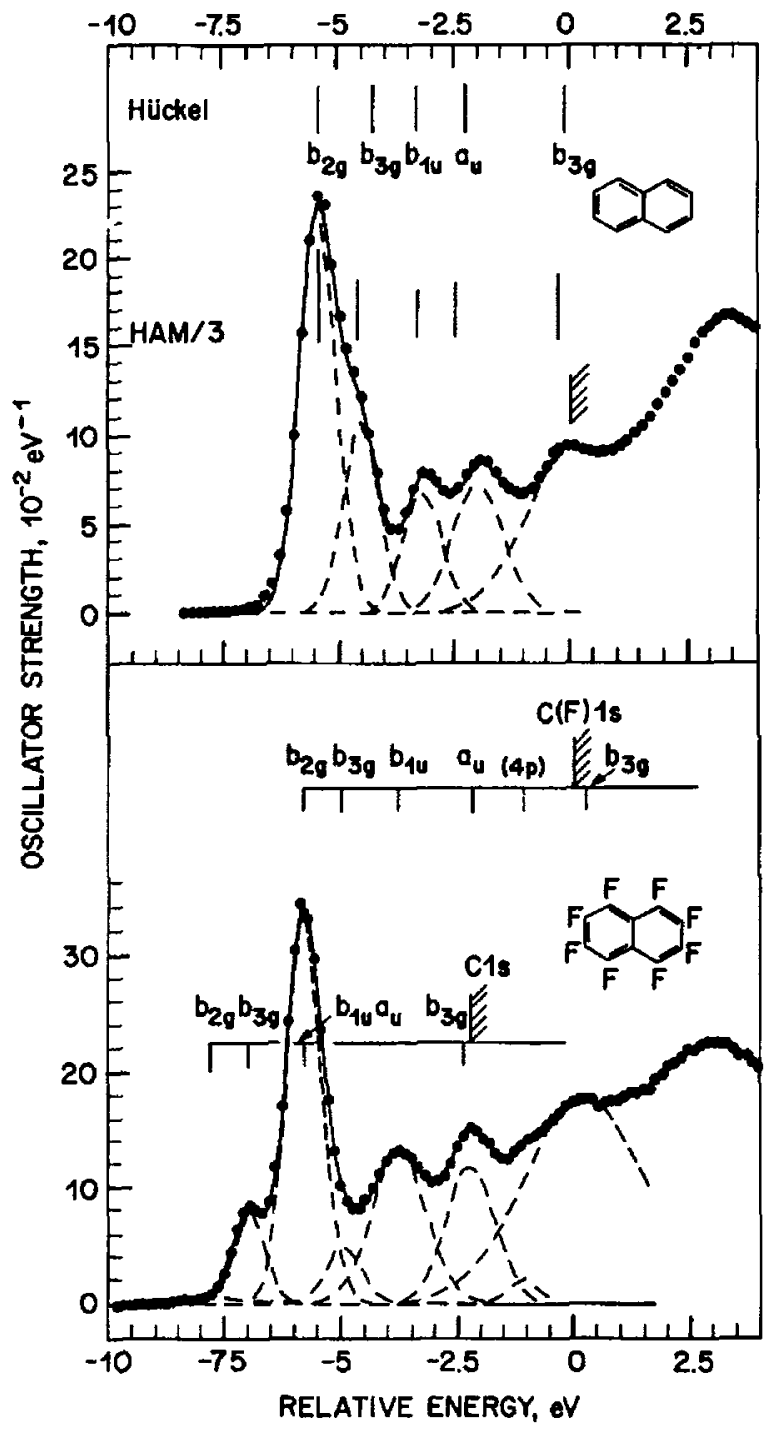

Fig. 9. Expansion of the $\mathrm{C} 1 s \rightarrow n \pi *$ regions of the naphthalene and octafluoronaphthalene spectra. The dashed curves are the result of spectral decomposition into Gaussian components using leastsquares curve fitting. Also shown are the $\pi^{*}$ virtual orbital energies of ground-state naphthalene calculated using the Hückel and HAM/3 [51, 52] methods. The heights of the HAM/3 lines represent predicted relative intensities based upon the squares of the $\mathrm{C} 2 p \pi$ coefficients in the calculated terminating MO's.

aligned with the first in the parent (Fig. 9), then a good match between the higher pre-edge features is observed; this implies that the four matching peaks in the octafluoro compound (bands $2,3,4$, and 6 ) are $\mathrm{C} 1 s(\mathrm{CF}) \rightarrow n \pi^{*}$ transitions, analogous to the $\mathrm{C} 1 s(\mathrm{CH}) \rightarrow n \pi^{*}$ bands of naphthalene, whereas band 1 of octafluoronaphthalene corresponds to a transition not observed in naphthalene. Our assignments for the $\mathrm{C} 1 s(\mathrm{C}) \rightarrow n \pi^{*}$ transitions in octafluoronaphthalene are based upon the expectation that the relative intensities of the $\mathrm{C} 1 s \rightarrow n \pi^{*}$ transitions will be proportional to the square of the LCAO coefficient of the $2 p \pi A O$ in the $n$th $M O$ at the site of the core excitation. Since both 
TABLE 6

Energies $(\Delta E, \mathrm{eV})$, term values (TV, $\mathrm{eV})$, oscillator strengths $\left(f, \times 10^{-2}\right)$ and proposed assignments in the $1 s$ spectra of naphthalene and octafluoronaphthalene

Carbon $1 s$

Naphthalene

Octafluoronaphthalene

\begin{tabular}{|c|c|c|c|c|c|c|c|c|c|c|c|c|}
\hline \multicolumn{4}{|l|}{$\mathrm{C} 1 s$} & \multicolumn{4}{|c|}{ Cls (C) } & \multicolumn{4}{|c|}{$\mathrm{Cls}$ (CF) } & \multirow{2}{*}{$\begin{array}{l}\text { Proposed } \\
\text { Assignment }\end{array}$} \\
\hline Band & $\Delta E^{\mathrm{a}}$ & $\mathrm{TV}$ & $f$ & Band & $\Delta E^{\mathbf{a}}$ & $\mathrm{TV}$ & $f^{\mathrm{b}}$ & Band & $\Delta E^{\mathrm{a}}$ & $\mathrm{TV}$ & $f^{b}$ & \\
\hline$\overline{14}$ & $284.95^{c}$ & 5.45 & 21 & & 285.4 & 6.0 & 0.8 & 2 & $287.4^{\mathrm{d}}$ & 6.2 & $(26)$ & $b_{2 g} \pi^{*}$ \\
\hline 2 & 285.83 & 4.57 & 10 & 1 & 286.2 & 5.2 & 6.7 & {$\left[2^{\prime}\right.$} & $\sim 288.2$ & $\sim 5.4]^{\mathrm{b}}$ & 4.7 & $b_{3 g} \pi^{*}$ \\
\hline 3 & 287.20 & 3.20 & 6 & 2 & 287.4 & 4.0 & $(6)$ & 3 & 289.4 & 4.2 & 20 & $b_{1 \mathrm{u}} \pi^{*} / \sigma^{*}(\mathrm{C}-\mathrm{F})$ \\
\hline 4 & 288.42 & 2.0 & 8 & & & & & 4 & 290.9 & 2.7 & (13) & $a_{u} \pi^{*} / \sigma^{*}(\mathrm{C}-\mathrm{F})$ \\
\hline & & & & 3 & 289.4 & 2.0 & & 5 & 292.1 & 1.5 & 1.7 & $4 p$ \\
\hline 5 & $\begin{array}{l}290.3 \\
290.4^{\mathrm{f}}\end{array}$ & 0.1 & $(10)^{e}$ & 4 & $\begin{array}{l}290.9 \\
291.4^{8}\end{array}$ & 0.5 & (3) & 6 & $\begin{array}{l}293.4 \\
293.6^{\mathrm{g}}\end{array}$ & 0.2 & - & $\begin{array}{l}b_{3 \mathrm{~g}} \pi^{*} \\
\mathrm{IP}\end{array}$ \\
\hline 6 & 293.6 & -3.2 & & & & & & 7 & 296.2 & -2.6 & & $\sigma *(\mathrm{C}-\mathrm{C})$ \\
\hline 7 & 300.2 & -10 & & 8 & 299.2 & -8.2 & & 8 & 299.2 & -5.6 & & $\sigma^{*}(\mathrm{C}-\mathrm{C})$ \\
\hline 8 & 305 & -15 & & 9 & 304 & -13 & & $\begin{array}{c}9 \\
10^{h}\end{array}$ & $\begin{array}{l}304 \\
327\end{array}$ & $\begin{array}{l}-10 \\
-34\end{array}$ & & $\begin{array}{l}\sigma^{*}(\mathrm{C}-\mathrm{C}) \\
\text { EXAFS? }\end{array}$ \\
\hline
\end{tabular}

Fluorine 1s

Octafluoronaphthalene

\begin{tabular}{llcl} 
Band & $\Delta E$ & TV & Proposed Assignment \\
\hline $1^{\mathrm{a}}$ & 689.0 & 4.6 & $b_{2 \mathrm{~g}} \pi^{*}, \sigma^{*}(\mathrm{C}-\mathrm{F})$ \\
2 & 691.7 & 1.9 & $\mathrm{a}_{\mathrm{u}} \pi^{*}, \sigma^{*}(\mathrm{C}-\mathrm{F})$ \\
& $693.6^{\mathrm{s}}$ & & $\mathrm{IP}$ \\
3 & $694.0(3)$ & -0.4 & $b_{3 \mathrm{~g}} \pi^{*}$ \\
4 & 697.0 & -3.4 & $\sigma^{*}(\mathrm{C}-\mathrm{C})$ \\
$5^{\mathrm{h}}$ & $727(2)$ & -33 & EXAFS \\
\hline
\end{tabular}

${ }^{a} \mathrm{Cls} \rightarrow n \pi *$ energies from curve fitting as in Fig. 9. 'Values in parentheses involve overlapping features originating at $\mathrm{C} 1 s(\mathrm{C})$ and $\mathrm{C} 1 s$ (CF). The total peak intensity in this case was divided, with $80 \%$ allocated to $\mathrm{C} 1 s$ (CF) and $20 \%$ to $\mathrm{C} 1 s$ (C). ${ }^{\circ}$ Calibrated as 116.1 (2) $\mathrm{eV}$ below the $\mathrm{N} 1 s \rightarrow 1 \pi^{*}$ transition of $\mathrm{N}_{2}$ at $401.1 \mathrm{eV}$. ${ }^{d}$ Calibrated as $3.39(2) \mathrm{eV}$ below the $\mathrm{C} 1 s \rightarrow 1 \pi^{*}$ transition in $\mathrm{CO}_{2}$ at $290.74 \mathrm{eV}$ [44].$^{\circ}$ Value poorly defined due to overlap with the C1s ionization continuum. 'Tonization potential estimated as equal to that of benzene [39], with no distinction between the ionization potentials of bridgehead and non-bridgehead carbons. ${ }^{\circ} \mathrm{C} 1 s(\mathrm{C})$, $\mathrm{C} 1 s$ (CF) and F1s ionization potentials assumed equal to those of 1, 2, 3,4-tetrafluorobenzene [39]. ${ }^{\text {hObserved }}$ in longer range scans; does not appear in Fig. 8.

the $b_{2 \mathrm{~g}} \pi^{*}$ and $a_{\mathrm{u}} \pi^{*}$ MO's of naphthalene and octafluoronaphthalene have coefficients at the bridgehead carbons which are exactly zero by symmetry [54], the transitions from the $\mathrm{C} 1 s(\mathrm{C})$ orbitals of the bridgehead atoms to these $\pi^{*}$ MO's should not be observed. Thus the lowest transition observed in octafluoronaphthalene $(286.2 \mathrm{eV})$ is assigned as $\mathrm{C} 1 s(\mathrm{C}) \rightarrow b_{3 \mathrm{~g}} \pi^{*}$ rather than to $\mathrm{C} 1 s(\mathrm{C}) \rightarrow b_{2 \mathrm{~g}} \pi^{*}$ as in naphthalene. Note however that curve fitting reveals a very weak feature at $285.4 \mathrm{eV}$ (see Fig. 9) which is possibly the forbidden $\mathrm{C} 1 s(\mathrm{C}) \rightarrow b_{2 \mathrm{~g}} \pi^{*}$ transition. This interesting complication in the inner-shell 
spectrum of octafluoronaphthalene does not appear in naphthalene itself since the excitations from $\mathrm{C} 1 s(\mathrm{C}-\mathrm{H})$ and $\mathrm{C} 1 s(\mathrm{C})$ cannot be resolved. Gaussian curve fitting was used to assist in identifying the $\mathrm{C} 1 s(\mathrm{CF}) \rightarrow b_{3 \mathrm{~g}} \pi^{*}$ and two other $\mathrm{C} 1 s$ (C) $\rightarrow n \pi^{*}$ transitions in octafluoronaphthalene which were expected to have nonzero intensity. From this (as well as term value arguments), it is concluded that the $\mathrm{C} 1 s(\mathrm{C}) \rightarrow b_{1 \mathrm{u}} \pi^{*}$ transition underlies feature 2 . According to the bond length versus $\sigma^{*}$ term value relationship [14], the C1s (CF) $\rightarrow$ $\sigma^{*}(\mathrm{C}-\mathrm{F})$ transitions are expected to have term values of $3-5 \mathrm{eV}$. The most likely candidates are features 3 and 4 , which are enhanced relative to the corresponding features in naphthalene. (Note that the sum of the $\mathrm{C} 1 s(\mathrm{C}) \rightarrow n \pi^{*}$ and $\mathrm{C} 1 s(\mathrm{CF}) \rightarrow n \pi^{*}$ intensities in octafluoronaphthalene are in reasonable agreement with those of naphthalene except for features 3 and 4).

The maxima in the first $15 \mathrm{eV}$ of the $\mathrm{C} 1 s$ continua of naphthalene and octafluoronaphthalene are attributed to $\mathrm{C} 1 s \rightarrow \sigma^{*}(\mathrm{C}-\mathrm{C})$ transitions. The pattern of two broad resonances observed in naphthalene is similar to that in benzene [19] and the $\mathrm{C} 1 s(\mathrm{CH})$ spectra of the fluorobenzenes [10], whereas the pattern of three maxima observed in octafluoronaphthalene parallels the pattern observed for transitions originating at $\mathrm{C} 1 s(\mathrm{CF})$ in the fluorobenzene continua [10].

Two broad bands are observed between 290 and $310 \mathrm{eV}$ in the NEXAFS of thin-film naphthalene [53]. The polarization dependence of these features is opposite to that of the $\mathrm{C} 1 s \rightarrow n \pi^{*}$ transitions, thus leading to $\mathrm{C} 1 s \rightarrow \sigma^{*}(\mathrm{C}-\mathrm{C})$ assignments for features $6-8$. We note that the maximum at $294 \mathrm{eV}$ is considerably weaker in the NEXAFS of the condensed naphthalene film than in the vapor-phase spectrum. This difference can be explained by contributions from a double excitation involving $\pi \rightarrow \pi^{*}$ shake up accompanying C1s $\rightarrow \pi^{*}$ excitation which is damped in the condensed phase. A related anomaly between the spectrum of free benzene and the NEXAFS of benzene films on $\mathrm{Cu}(100)$ [55] or Pt (111) [19] has been explained in terms of variable environment-dependent contributions of $\pi$-symmetry double excitations using ab initio multiconfiguration calculations [56].

Being planar systems, the naphthalene/octafluoronaphthalene pair follow the perfluoro rule in regard of their $n \pi(n=1-5)$ valence ionization potentials, Table 7 [6]. These splittings among the $n \pi$ levels are relevant to the ISEELS spectra, for the $n \pi^{*}$ levels and thus the $\mathrm{C} 1 s \rightarrow n \pi^{*}$ transitions of naphthalene are expected to show the same splitting pattern as the $n \pi$ levels according to the pairing theorem (see below). If the perfluoro effect applies to the innershell excited configurations, the $n \pi^{*}$ splitting pattern of naphthalene will also be seen in the $\mathrm{C} 1 s$ spectra of octafluoronaphthalene, with $\left(\mathrm{C} 1 s, n \pi^{*}\right)$ term values closely similar in the two molecules.

The $n \pi$ and $n \pi^{*}$ orbital energies and TNI data are summarized in Table 7. One sees from this data that there is quite good agreement of the various $n \pi^{*}$ level energies as deduced from photoelectron spectroscopy (PES) and IS- 
TABLE 7

Intervals $(\mathrm{eV})$ in the $n \pi$ and $n \pi^{*}$ manifolds of naphthalene and octafluoronaphthalene

\begin{tabular}{|c|c|c|c|c|c|c|c|c|c|}
\hline \multicolumn{4}{|c|}{$n \pi(\mathrm{PES})^{a}$} & \multicolumn{6}{|l|}{$n \pi^{*}$} \\
\hline \multirow[t]{2}{*}{ sym $^{b}$} & \multirow[t]{2}{*}{$n$} & \multirow[t]{2}{*}{$\mathrm{C}_{10} \mathrm{H}_{8}$} & \multirow[t]{2}{*}{$\mathrm{C}_{10} \mathrm{~F}_{8}$} & \multicolumn{4}{|c|}{$\mathrm{C}_{10} \mathrm{H}_{8}$} & \multicolumn{2}{|l|}{$\mathrm{C}_{10} \mathrm{~F}_{8}$} \\
\hline & & & & sym & $n$ & $\mathrm{C} 1 s \rightarrow n \pi^{*}$ & $\mathrm{TNI}^{\mathrm{c}}$ & $\mathrm{C} 1 s(\mathrm{C}) \rightarrow n \pi^{*}$ & $\mathrm{C} 1 s(\mathrm{CF}) \rightarrow n \pi^{*}$ \\
\hline$a_{u}$ & 1 & 0 & 0 & $b_{2 g}$ & 1 & 0 & 0 & {$[0]^{d}$} & 0 \\
\hline$b_{1 \mathrm{u}}$ & 2 & 0.75 & 1.03 & $b_{3 g}$ & 2 & 0.92 & 0.56 & $(0.8)$ & 0.82 \\
\hline$b_{3 g}$ & 3 & 1.88 & 1.91 & $b_{1 \mathrm{u}}$ & 3 & 2.22 & 1.15 & 2.0 & $2.01^{\circ}$ \\
\hline$b_{2 \mathrm{~g}}$ & 4 & 2.77 & 2.71 & $a_{u}$ & 4 & 3.43 & 3.20 & $-^{f}$ & 3.54 \\
\hline$b_{1 \mathrm{u}}$ & 5 & 4.37 & 4.66 & $b_{3 g}$ & 5 & 5.45 & $5.29^{\mathrm{g}}$ & 5.5 & 5.99 \\
\hline
\end{tabular}

aFrom the photoelectron spectra [6]. 'Symmetry representation in $D_{2 h} ; z$-axis is perpendicular to the molecular plane and the $x$-axis is the long-axis in-plane direction. 'Temporary negative ion resonances summarized in ref. [1]. ${ }^{d}$ Since the $\mathrm{C} 1 s(\mathrm{C}) \rightarrow b_{2 \mathrm{~g}} \pi^{*}$ transition is expected to have zero intensity, this reference level cannot be determined accurately. The $\mathrm{C} 1 s(\mathrm{C}) \rightarrow b_{3 \mathrm{~g}} \pi^{*}$ transition is assumed to be $0.8 \mathrm{eV}$ above that of $\mathrm{Cls}(\mathrm{C}) \rightarrow b_{2 \mathrm{~g}} \pi^{*}$ to approximate this reference level. ${ }^{\circ} \mathrm{From}$ Gaussian curve fitting (see Fig. 9). ${ }^{\mathrm{T}} \mathrm{The} \mathrm{Cls}(\mathrm{C}) \rightarrow a_{1} \pi^{*}$ transition is expected to have zero intensity because of the lack of $2 p \pi$ density on the bridgehead carbon atoms (C). ${ }^{8}$ Possibly due to a Feshbach resonance.

EELS data, and that the TNI data for naphthalene [1] also fits this picture rather well. Looking at the term values of the $\mathrm{C} 1 s \rightarrow 1 \pi^{*}$ transitions, that of naphthalene $(5.45 \mathrm{eV})$ is matched fairly well by that of octafluoronaphthalene [6.2 eV for the transition originating at $\mathrm{C} 1 s(\mathrm{CF})$ ], while that for (F1s, $\left.1 \pi^{*}\right)$ is again somewhat smaller $(4.6 \mathrm{eV})$ than observed for excitations originating at $\mathrm{C} 1 s$. The term values of the higher energy $\mathrm{C} 1 s \rightarrow n \pi^{*}$ transitions of naphthalene and octafluoronaphthalene also agree to better than $1 \mathrm{eV}$.

One concludes that the perfluoro effect holds for the $n \pi^{*}$ levels of octafluoronaphthalene. On this basis, one is hopeful also of finding a transition to $\sigma^{*}$ among those to $\pi^{*}$ in octafluoronaphthalene, for this is the situation in hexafluorobenzene [10] and tetrafluoroethylene. However, the evidence for $\mathrm{C} 1 s \rightarrow \sigma^{*}(\mathrm{C}-\mathrm{F})$ transitions is very weak in the present case, probably because of the greater density of overlapping $1 s \rightarrow n \pi^{*}$ transitions in the expected energy region of the larger molecules. The second feature in the F1s spectrum of octafluoronaphthalene is quite intense, and probably corresponds to $\mathrm{F} 1 s \rightarrow \sigma^{*}(\mathrm{C}-\mathrm{F})$, although excitations to higher lying $\pi^{*} \mathrm{MO}$ 's also will contribute here.

Acetic Acid

Acetic acid is another unsaturated system in which perfluorination of the methyl group is expected to lead to a large increase in the $\left(\mathrm{C} 1 s, 1 \pi^{*}\right)$ term 


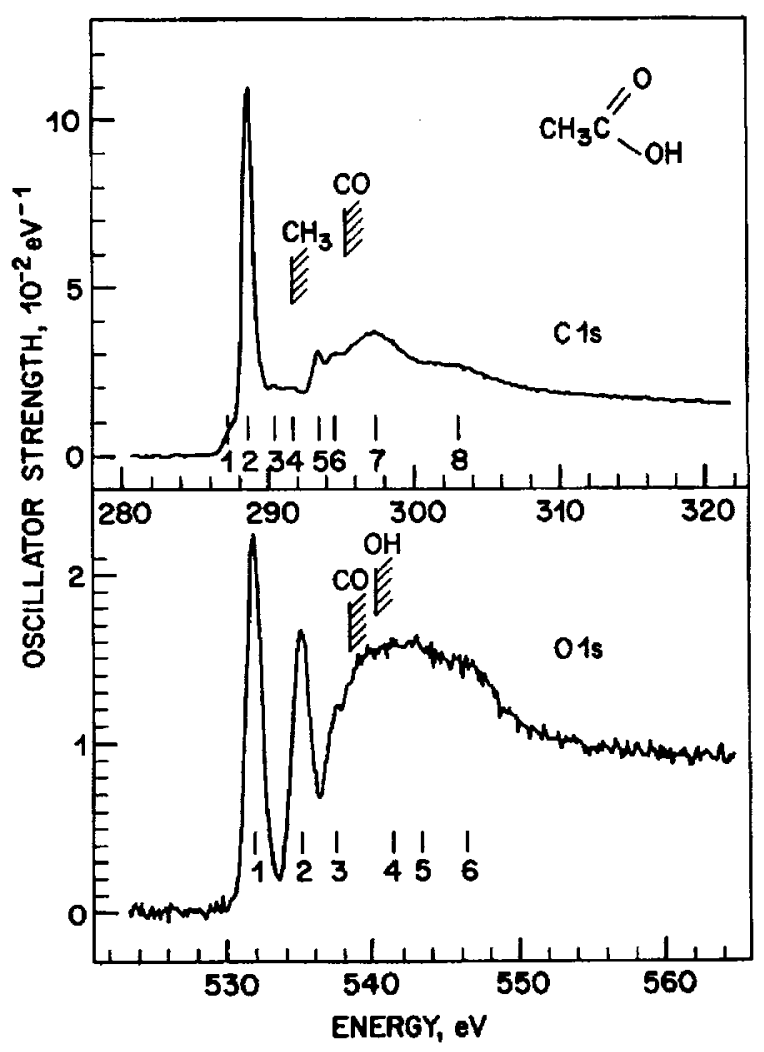

Fig. 10. C1s and O1s oscillator strength distributions derived from the ISEEL spectra of acetic acid. Ionization potentials are indicated by the left-hand edges of the hatched areas.

value. The inner-shell oscillator strength spectra of acetic acid and trifluoroacetic acid appear in Figs. 10 and 11, and are tabulated in Tables 8 and 9. As expected, the $\left(\mathrm{C} 1 s, 1 \pi^{*}\right)$ term value of trifluoroacetic acid is quite large, being $8.1 \mathrm{eV}$ for the carboxyl $\mathrm{C}$ atom while the $\left(01 \mathrm{~s}, 1 \pi^{*}\right)$ term value amounts to 7.7 $\mathrm{eV}$ for the carbonyl $\mathrm{O}$ atom. The term values for excitation to $1 \pi^{*}$ from $\mathrm{C} 1 s(\mathrm{C}=0)$ and $01 s(\mathrm{C}=0)$ in acetic acid on the other hand are only 6.9 and $6.3 \mathrm{eV}$, respectively, $1,2-1.4 \mathrm{eV}$ smaller than observed for the trifluoro derivative. Thus perfluorination of acetic acid has nearly the same effect on the $\left(\mathrm{C} 1 s(\mathrm{C}=0), 1 \pi^{*}\right)$ and $\left(01 s(\mathrm{C}=0), 1 \pi^{*}\right)$ term values, increasing them significantly over their values in the protio species, as appropriate for nonplanar systems.

As experienced in both hexafluoroacetone and hexafluorobutyne-2, the $\mathrm{F} 1 s \rightarrow 1 \pi^{*}$ excitation in trifluoroacetic acid has a significantly smaller term value than $\mathrm{C} 1 s \rightarrow 1 \pi^{*}$; the (F1s, $\left.1 \pi^{*}\right)$ term values in these three compounds are 5.9 , 4.6 and $5.0 \mathrm{eV}$, respectively. The generally smaller term value observed for $\mathrm{F} 1 s \rightarrow 1 \pi^{*}$ excitations is felt to be a consequence of the atom-to-atom charge transfer inherent in this transition. This effect also is apparent in the term values for $\mathrm{O} 1 s(\mathrm{OH}) \rightarrow 1 \pi^{*}$, which are 1.4-1.8 eV smaller than those of $01 s(\mathrm{C}=0) \rightarrow 1 \pi^{*}$ in acetic acid and trifluoroacetic acid. 


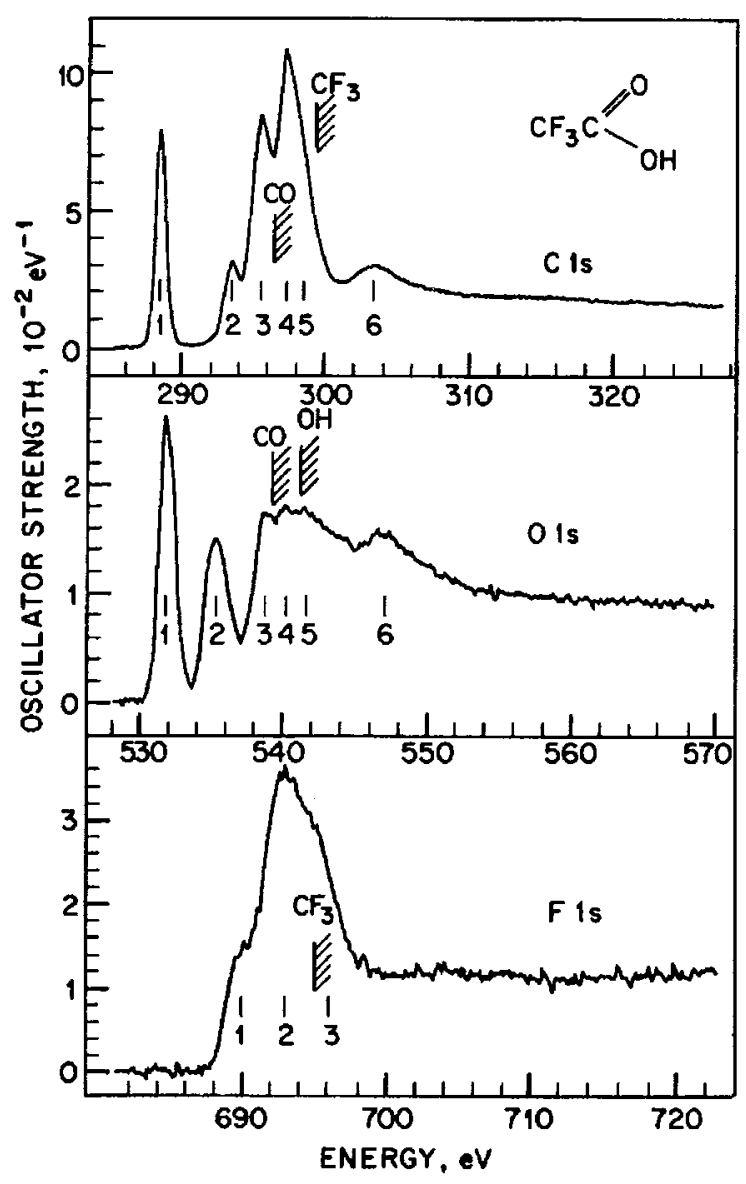

Fig. 11. C1s, $\mathrm{O} 1 s$ and $\mathrm{F} 1 s$ oscillator strength distributions derived from the ISEEL spectra of trifluoroacetic acid. Ionization potentials are indicated by the left-hand edges of the hatched areas.

The higher-energy features in the inner-shell spectra of the carboxylic acids are assigned to excitations to Rydberg and $\sigma^{*}$ MO's (Tables 8 and 9 ), based upon their term values [1] and the bond length correlation [14]. The C1s and $01 s$ spectra of acetic acid are very similar to those of propionic acid [57]. In particular, weak $\mathrm{C} 1 s(\mathrm{CH}) \rightarrow 3 s / \sigma^{*}$ transitions precede the more intense $\mathrm{C} 1 s(\mathrm{C}=0) \rightarrow 1 \pi^{*}$ transitions in both molecules. The spectrum of trifluoroacetic acid shows the characteristic intense transitions from $\mathrm{C} 1 s$ and F1s to the $\sigma^{*}(\mathrm{C}-\mathrm{F})$ levels of the $\mathrm{CF}_{3}$ group. Similar $\mathrm{CF}_{3}$-related structures are observed in hexafluoroacetone, hexafluorobutyne-2 (vide supra), trifluoromethyl hypofluorite [2], bis-trifluoromethyl peroxide [2], and many perfluoroalkanes [3]. The pattern of two intense peaks separated by $3.5 \mathrm{eV}$ in the $01 s$ spectra of both acetic and trifluoroacetic acids is characteristic of the carboxylic acid functional group [57].

\section{Acetonitrile}

By analogy with the trifluoromethyl systems discussed above, one expects a significant increase in the $\left(\mathrm{N} 1 s, 1 \pi^{*}\right)$ and $\left(\mathrm{C} 1 s(\mathrm{CN}), 1 \pi^{*}\right)$ term values on 
TABLE 8

Energies $(\Delta E, \mathrm{eV})$, term values $(\mathrm{TV}, \mathrm{eV})$ and proposed assignments of the $1 s$ spectra of acetic acid

\begin{tabular}{|c|c|c|c|c|c|}
\hline \multicolumn{2}{|c|}{ Carbon $1 s$} & \multicolumn{2}{|l|}{ TV } & \multicolumn{2}{|l|}{ Assignment } \\
\hline Band & $\Delta E$ & $\left(\mathrm{CH}_{3}\right)$ & $(\mathrm{C}=0)$ & $\mathrm{CH}_{3}$ & $\mathrm{C}=0$ \\
\hline 1 & 287.4 & 4.6 & & $3 s$ & \\
\hline 2 & $288.69^{\mathrm{a}}$ & 2.9 & 6.9 & $3 p / \sigma^{*}(\mathrm{C}-\mathrm{H})$ & $1 \pi^{*}$ \\
\hline 3 & 290.3 & 1.3 & & $4 p$ & \\
\hline \multirow[t]{2}{*}{4} & 291.5 & & 4.1 & & $3 s$ \\
\hline & $291.6^{\mathrm{b}}$ & & & IP & \\
\hline 5 & 293.4 & -1.8 & 2.2 & $\sigma^{*}(\mathrm{C}-\mathrm{C})$ & $3 p$ \\
\hline \multirow[t]{2}{*}{6} & 294.6 & & 1.0 & & $4 p$ \\
\hline & $295.6^{\mathrm{b}}$ & & & & IP \\
\hline 7 & 297.3 & & -1.7 & & $\sigma^{*}(\mathrm{C}-\mathrm{C})$ \\
\hline 8 & $303.1(5)$ & & -7.5 & & $\sigma^{*}(\mathrm{C}=\mathrm{O})$ \\
\hline
\end{tabular}

Oxygen 1s

\begin{tabular}{|c|c|c|c|c|c|}
\hline \multirow[b]{2}{*}{ Band } & \multirow[b]{2}{*}{$\Delta E$} & \multicolumn{2}{|l|}{ TV } & \multicolumn{2}{|c|}{ Assignment } \\
\hline & & $(\mathrm{CO})$ & $(\mathrm{OH})$ & $\mathrm{Co}$ & $\mathrm{OH}$ \\
\hline 1 & $531.95^{c}$ & 6.3 & & $1 \pi^{*}$ & \\
\hline 2 & 535.2 & 3.1 & 4.9 & $3 p$ & $1 \pi^{*}$ \\
\hline 3 & $\begin{array}{l}537.6 \\
538.3^{\text {b }}\end{array}$ & 0.7 & 2.5 & $\begin{array}{l}\text { Ryd } \\
\text { IP }\end{array}$ & $3 p / \sigma^{*}(\mathrm{O}-\mathrm{H})$ \\
\hline & $540.1^{b}$ & & & & IP \\
\hline 4 & $541.4(8)$ & -3.1 & -1.3 & $\sigma^{*}(\mathrm{C}-\mathrm{O})$ & $\sigma^{*}(\mathrm{C}-\mathrm{C})$ \\
\hline 5 & $543.5(8)$ & & -3.4 & & $\sigma^{*}(\mathrm{C}-\mathrm{O})$ \\
\hline 6 & $546.3(8)$ & -8.0 & & $\sigma^{*}(\mathrm{C}=0)$ & \\
\hline
\end{tabular}

aCalibrated as $2.05(5) \mathrm{eV}$ below the $\mathrm{C} 1 s \rightarrow 1 \pi^{*}$ transition of $\mathrm{CO}_{2}$ at $290.74 \mathrm{eV}$. brom ref. 39 . ${ }^{\circ}$ Calibrated as $2.26(5) \mathrm{eV}$ below the $\mathrm{O} 1 s \rightarrow 1 \pi^{*}$ transition of $\mathrm{CO}$ at $534.21 \mathrm{eV}$.

going from acetonitrile to trifluoroacetonitrile. In acetonitrile, the term values in question are 6.2 and $6.0 \mathrm{eV}$, respectively [58]. Although the vapor-phase spectra of $\mathrm{CF}_{3} \mathrm{CN}$ have not been studied yet, the NEXAFS spectrum of solid $\mathrm{CF}_{3} \mathrm{CN}$ is available [59]. Because of the remarkable similarity of the innershell spectra of the free $\mathrm{CH}_{3} \mathrm{CN}$ molecule [58] and the solid [59], it is thought that the NEXAFS spectrum of solid $\mathrm{CF}_{3} \mathrm{CN}$ will be a good approximation to the free-molecule spectrum. Using estimated vapor-phase ionization potentials of $294 \mathrm{eV}(\mathrm{Cl} s(\mathrm{CN}))$ and $406 \mathrm{eV}(\mathrm{N} 1 s)$ for $\mathrm{CF}_{3} \mathrm{CN}$ leads to $(\mathrm{C} 1 s(\mathrm{CN})$, $\left.1 \pi^{*}\right)$ and $\left(\mathrm{N} 1 s, 1 \pi^{*}\right)$ term values of 8 and $7 \mathrm{eV}$, respectively. Again one observes a significant increase of the term values for transitions terminating at $1 \pi^{*}$ upon perfluorination, consistent with the inductive shift of the $1 \pi^{*}$ orbital energy by the out-of-plane $\mathrm{F}$ atoms. 


\section{TABLE 9}

Energies $(\Delta E, \mathrm{eV})$, term values $(\mathrm{TV}, \mathrm{eV})$ and proposed assignments of the $1 s$ spectra of trifluoroacetic acid

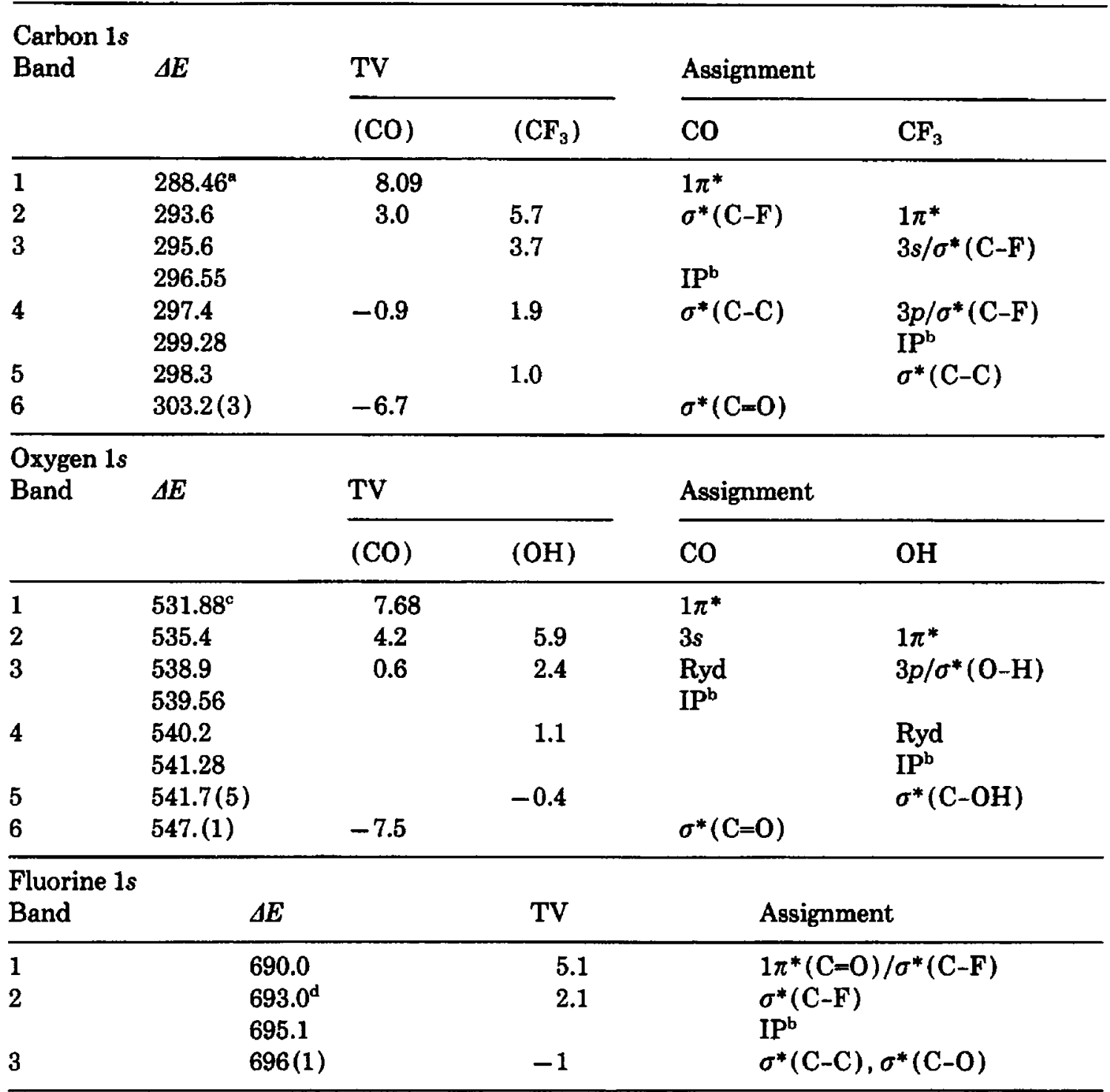

${ }^{a}$ Calibrated as $1.06(3) \mathrm{eV}$ above the $\mathrm{C} 1 s \rightarrow 1 \pi^{*}$ transition of $\mathrm{CO}$ at $287.40 \mathrm{eV}$. ${ }^{\mathrm{b}} \mathrm{From} \mathrm{X}$-ray photoelectron spectra [39] . 'Calibrated as $2.33(3) \mathrm{eV}$ below the $01 s \rightarrow 1 \pi^{*}$ transition of $\mathrm{CO}$ at 534.21 $\mathrm{eV} .{ }^{\mathrm{d} C}$ Calibrated as $158.8(2) \mathrm{eV}$ above the $\mathrm{O} 1 s \rightarrow 1 \pi^{*}$ transition of $\mathrm{CO}$ at $534.21 \mathrm{eV}$.

\section{INNER-SHELL OSCILLATOR STRENGTHS}

The oscillator strengths of the $\mathrm{C} 1 s \rightarrow 1 \pi^{*}$ and $\mathrm{O} 1 s \rightarrow 1 \pi^{*}$ transitions of the molecules considered in this work are summarized in Table 10. As was observed in previous studies of the fluorobenzenes [10] and fluoroethylenes [20], substitution of hydrogen by fluorine increases the $1 s \rightarrow 1 \pi^{*}$ oscillator strengths. This intensity effect is larger the closer the fluorines are situated to the unsat- 
urated center, and is also larger in alkenes and alkynes where the fluorines are bonded to both ends of the unsaturation, than in carbonyls where the fluorines are bonded to only one end. The intensification due to fluorination is apparent as well in the spectra of the partially fluorinated ethylenes, Fig. $2 b$, where it is seen that of the two transitions to $1 \pi^{*}$ in each molecule, the stronger is that originating at the $\mathrm{C}$ atom bonded to the larger number of $\mathrm{F}$ atoms. The intensification induced by fluorine can be explained in terms of a radial contraction of the $\pi^{*} \mathrm{MO}$ on the fluorinated carbon atoms which in turn leads to a larger $\langle 1 s|\mu| 2 p \pi\rangle$ transition density. The contraction can be rationalized either in terms of the potential barrier effect [60], or in terms of the inductive effect of the electronegative $\mathrm{F}$ atoms [20].

Intensification upon fluorination also is observed in the $\mathrm{C} 1 s$ spectra of the alkanes [3], although in this case the transitions terminate at $\sigma^{*}(\mathrm{C}-\mathrm{F})$ rather than at $1 \pi^{*}$. The much larger intensity of transitions to $\sigma^{*}(\mathrm{C}-\mathrm{F})$ as compared with those to $\sigma^{*}(\mathrm{C}-\mathrm{H})$ is evident on comparison of the spectra of the fluoroethylenes (Fig. 2), octafluorocyclopentene (Fig. 3), hexafluorobutyne-2 (Fig. 7 ) and trifluoroacetic acid (Figs. 10,11) with their perhydrogenated parents. In the latter three species and in hexafluoroacetone, $\mathrm{C} 1 s \rightarrow \sigma^{*}(\mathrm{C}-\mathrm{F})$ transitions have even greater oscillator strengths than the $\mathrm{C} 1 s \rightarrow 1 \pi^{*}$ features even after corrections for orbital degeneracies. The systematic increase in $\mathrm{C} 1 s \rightarrow \sigma^{*}(\mathrm{C}-\mathrm{F})$ oscillator strength with increasing fluorination has been documented and discussed recently [61].

It is interesting to note that the $\mathrm{O} 1 s \rightarrow 1 \pi^{*}(\mathrm{CO})$ oscillator strength decreases with fluorination in the series $\mathrm{H}_{2} \mathrm{CO}, \mathrm{HFCO}, \mathrm{F}_{2} \mathrm{CO}$, suggesting an increasing polarization of the $1 \pi^{*}$ MO away from the oxygen atoms with fluorination. Simultaneously, the $\mathrm{C} 1 s \rightarrow 1 \pi^{*}$ oscillator strength increases with fluorination such that the sums of the $\mathrm{C} 1 s \rightarrow 1 \pi^{*}$ and $01 s \rightarrow 1 \pi^{*}$ oscillator strengths are nearly constant. The fluorine-induced $1 \pi^{*}$ polarizations implied by these trends in $1 s \rightarrow 1 \pi^{*}$ oscillator strength are evident in the $1 \pi^{*}$ wavefunctions calculated for the ground states of these molecules [62], indicating that inner-shell excitation intensities can be used at least qualitatively to probe the spatial distributions of ground-state virtual orbitals [21]. The effect of methylation on $1 \pi^{*}$ of formaldehyde is opposite to that of fluorination, with the $\mathrm{C} 1 s \rightarrow 1 \pi^{*}$ oscillator strength in acetone being somewhat smaller than that in formaldehyde.

Comparison of the inner-shell oscillator strengths in butene-2 and butyne2 should reveal the double degeneracy of $1 \pi^{*}$ in the latter. Similarly, the strength of the transition to $1 \pi^{*}$ should be twice as large in $\mathrm{CF}_{3} \mathrm{CCCF}_{3}$ as in $\mathrm{CF}_{3} \mathrm{CFCFCF}_{3}$. The measured ratio in each of these two cases is 1.7, Table 10, which is similar to the ratio of 1.9 between the $\mathrm{C} 1 s \rightarrow 1 \pi^{*}$ transitions of acetylene and ethylene and the ratio of 1.8 between the related transitions in acrylic and propiolic acids [57].

The C1s spectrum of ethylene, Fig. 2a, is of interest for it shows the simultaneous presence of electronically allowed $\mathrm{C} 1 s \rightarrow 1 \pi^{*}$ and $\mathrm{C} 1 s \rightarrow 3 p_{\mathrm{xyz}}$ transi- 
TABLE 10

Oscillator strengths $\left(\times 10^{-2}\right)$ of $1 s \rightarrow 1 \pi^{*}$ transitions

\begin{tabular}{lcl}
\hline Planar Molecules & $f\left(\mathrm{C} 1 s \rightarrow 1 \pi^{*}\right)$ & $f\left(\mathrm{O} 1 s \rightarrow 1 \pi^{*}\right)$ \\
\hline $\mathrm{C}_{2} \mathrm{H}_{2}$ & 13.0 & \\
$\mathrm{C}_{2} \mathrm{H}_{4}$ & 6.8 & \\
$\mathrm{C}_{2} \mathrm{~F}_{4}$ & 11.3 & \\
cyclo- $\mathrm{C}_{5} \mathrm{H}_{8}$ & 8.0 & \\
cyclo- ${ }_{5} \mathrm{~F}_{8}$ & 12.1 & 7.2 \\
$\mathrm{H}_{2} \mathrm{CO}$ & 6.6 & 4.5 \\
$\mathrm{HFCO}$ & 10.4 & 2.6 \\
$\mathrm{~F}_{2} \mathrm{CO}$ & 13.2 & \\
$\mathrm{C}_{6} \mathrm{H}_{6}$ & 18.7 & \\
$\mathrm{C}_{6} \mathrm{~F}_{6}$ & 33.7 & \\
$\mathrm{C}_{10} \mathrm{H}_{8}$ & 22.3 & \\
$\mathrm{C}_{10} \mathrm{~F}_{8}$ & 32.5 & \\
$\mathrm{Nonplanar} \mathrm{Molecules}_{3}$ & & \\
$\mathrm{CH}_{3} \mathrm{CHCHCH}_{3}$ & $7.4^{\mathrm{a}}$ & \\
$\mathrm{CF}_{3} \mathrm{CFCFCF}_{3}$ & 9.7 & \\
$\mathrm{CH}_{3} \mathrm{CCCH}$ & \\
$\mathrm{CF}_{3} \mathrm{CCCF}_{3}$ & 12.6 & \\
$\left(\mathrm{CH}_{3}\right)_{2} \mathrm{CO}$ & 16.6 & \\
$\left(\mathrm{CF}_{3}\right)_{2} \mathrm{CO}_{\mathrm{CH}_{3} \mathrm{CO}} \mathrm{H}$ & $5.8^{\mathrm{b}}$ & 3.1 \\
$\mathrm{CF}_{3} \mathrm{CO}_{2} \mathrm{H}$ & $6.9^{\mathrm{b}}$ & 5.6 \\
\hline
\end{tabular}

${ }^{a}$ Average of cis-butene-2 (6.6) and trans-butene-2 (8.2 eV). ${ }^{b}$ Refers only to $\mathrm{C} 1 s(\mathrm{CO}) \rightarrow 1 \pi^{*}$ transition. 'Refers only to $\mathrm{O} 1 s(\mathrm{CO}) \rightarrow 1 \pi^{*}$ transition.

tions as well as the formally forbidden $\mathrm{C} 1 s \rightarrow 3 s$ band. Because the overlap density is larger between $1 s$ and $2 p \pi$ than between $1 s$ and $3 p$, the $1 s \rightarrow 1 \pi^{*}$ transition is more intense than $1 s \rightarrow 3 p$, the intensity factor being ca. 2.5. In turn, the $1 s \rightarrow 3 p$ transition is ca. 3 times more intense than $1 s \rightarrow 3 s$ in ethylene. These factors carry over to benzene, where the transition from $\mathrm{C} 1 s$ to $1 \pi^{*}$ is ca. 10 times stronger than that to $3 s$ [10]. Generalizing, it appears that oscillator strengths to $3 s$ from $\mathrm{C} 1 s$ in hydrocarbons will be smaller than $2.5 \times 10^{-2}$ in the largest of these. For comparison, the $\mathrm{O} 1 s \rightarrow 3 s$ transition of water has an oscillator strength of $1.4 \times 10^{-2}$ [2]. Note however, that these weak transitions to $3 s$ can be mixed with those to $\sigma^{*}$ having oscillator strengths of ca. $10 \times 10^{-2}$ [2] and thereby intensified appreciably.

\section{VARIATION OF $\left(1 s, 1 \pi^{*}\right)$ TERM VALUES WITH CHAIN LENGTH}

The effects of vinylogous substitution on $\pi$-electron spectral properties are very well known [63]. On going along the series from ethylene to butadiene, 


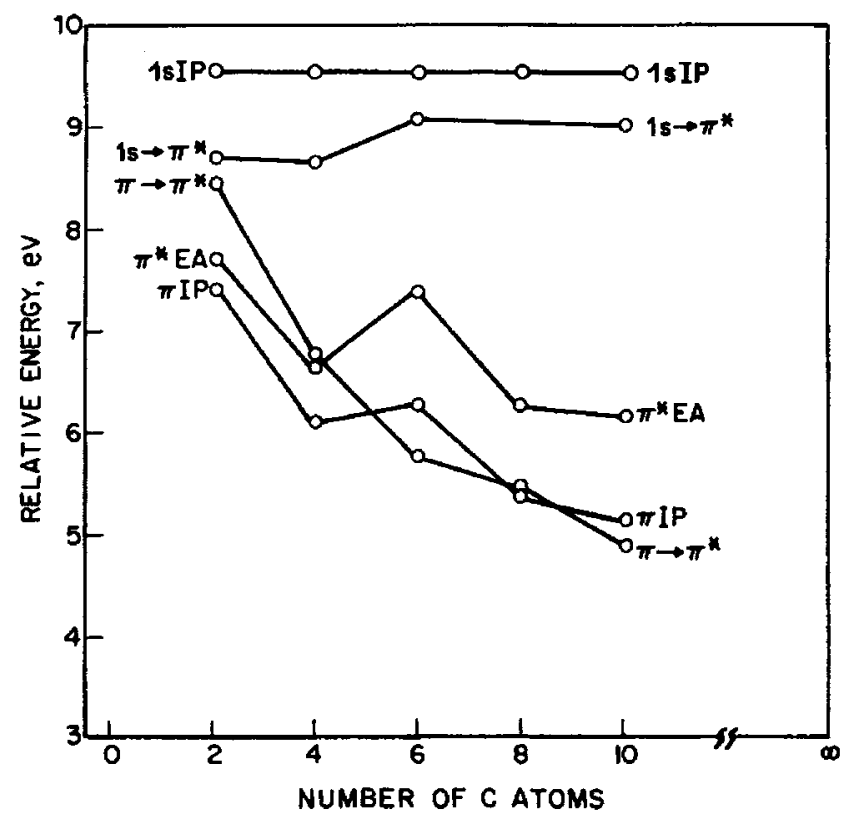

Fig. 12. Trends in $1 \pi$ and $1 \pi^{*}$ energies in a vinylogous series. Data are plotted for ethylene, butadiene, benzene, styrene and naphthalene. The $\mathrm{C} 1 s \rightarrow 1 \pi^{*}$ energy value plotted here is the absolute measured energy less $276.0 \mathrm{eV}$. The $\mathrm{C} 1 s$ ionization potential plotted is the absolute value less 282 $\mathrm{eV}$.

hexatriene, benzene, styrene and naphthalene, the energy of the highest occupied $\pi \mathrm{MO}, 1 \pi$, decreases as shown for the $1 \pi$ ionization potentials in Fig. 12. Because these systems are alternate, the $1 \pi$ and $1 \pi^{*}$ MO's are paired; thus vinylogous substitution not only raises the $1 \pi \mathrm{MO}$, but also lowers $1 \pi^{*}[26]$. This pairing is evident in Fig. 12, where $1 \pi^{*}$ electron affinities have also been plotted. As a consequence of the convergence of $1 \pi$ and $1 \pi^{*}$ energies with increasing size, the energy of the lowest $1 \pi \rightarrow 1 \pi^{*}$ transition also decreases (Fig. 12 ) as the size of the $\pi$ system increases.

Given this indisputable particle-in-a-box type behavior of the $1 \pi$ and $1 \pi^{*}$ MO energies, and the one-particle nature of the excitations, it is interesting to graph the $\mathrm{C} 1 s \rightarrow 1 \pi^{*}$ excitation energies in the same series of compounds. Assuming a constant $\mathrm{C} 1 s$ ionization potential in these compounds all of which consist of $\mathrm{C}$ atoms in $s p^{2}$ hybridization, one expects a rather sharp drop of the $\mathrm{C} 1 s \rightarrow 1 \pi^{*}$ excitation energy as the $\pi$-electron system is lengthened. In fact, the trend of the $\mathrm{C} 1 s \rightarrow 1 \pi^{*}$ excitation energies (plotted relative to an arbitrary reference energy of $276 \mathrm{eV}$ in Fig. 12) with increasing size is not steeply down, but in fact is slightly upward! Similarly, if we introduce the experimental C1s ionization potentials and then consider the $\left(\mathrm{C} 1 s, 1 \pi^{*}\right)$ term values, the expectation is that this quantity will increase with increasing extent of the $\pi$-electron system, whereas in fact it decreases slightly (from $6.2 \mathrm{eV}$ in ethylene to $5.4 \mathrm{eV}$ in naphthalene). 
The near-constancy of the $\left(\mathrm{C} 1 s, 1 \pi^{*}\right)$ term value with increasing molecular extent suggests that the form of the $1 \pi^{*}$ MO in unsaturated systems is inherently different when the excitation originates at $\mathrm{C} 1 s$. In fact, the critical element here is that the $\mathrm{C} 1 s$ hole in the $\left(\mathrm{C} 1 s, 1 \pi^{*}\right)$ configuration is localized (the $1 s-1 s$ splitting is believed to be of the order $10 \mathrm{meV}$ or so and certainly is less than $50 \mathrm{meV}$ based upon high resolution ISEELS [32]). Because of this localization, the potential experienced by the electron in $1 \pi^{*}$ in the short-lived $\left(\mathrm{C} 1 s, 1 \pi^{*}\right)$ configuration is significantly different from what it would be in the $\left(1 \pi, 1 \pi^{*}\right)$ configuration, for example.

Consider a conjugated $\pi$-electron system as viewed from the valence bond perspective. The ground state is represented by structure $A$, whereas the lowest-energy inner-shell excitation is represented by structure $B$. In this latter, the excitation site is denoted by $C\left(1 s^{-1}\right)$ and carries two electrons in its $2 p \pi$ AO. From the valence electron's perspective, the loss of a $1 s$ electron at $C$ is equivalent to replacing that atom by $\mathrm{N}$, as in structure $C$. We propose that structure $B$ (related to structure $C$ ) is especially stable, and is the only significant contributor to the $\left(\mathrm{C} 1 s, 1 \pi^{*}\right)$ upper state, with all other structures such as $D$ being charge separated and therefore of higher energy. Viewed in this way, the $\left(\mathrm{C} 1 s, 1 \pi^{*}\right)$ term value is then the $2 p \pi$ atomic ionization potential at the pseudo- $\mathrm{N}$ atom, and thus is independent of the molecular size of a planar system. In as much as the ( $\left.\mathrm{C} 1 s, 1 \pi^{*}\right)$ term value is near-constant in a vinylogous series and also behaves appropriately in regard to the perfluoro effect, one sees that the $\left(\mathrm{C} 1 s, 1 \pi^{*}\right)$ term value in all planar perfluorinated $\pi$-electron hydrocarbons regardless of size will be in the $5.5-6.5 \mathrm{eV}$ range.<smiles>C=CC=CC=C</smiles>

(A)<smiles>C=CCCCC=C</smiles>

(B)<smiles>C=CCNC=C</smiles>

(C)<smiles>C=C[C]=C[C]C</smiles>

(D)

Higher (C1s, $n \pi^{*}$ ) excited states such as structure $D$ are formed by localizing the $\mathrm{C} 1 s$ hole at site $q$ and transferring the electron to $2 p \pi$ on centers $q+2$, $q+4, q+6$, etc. As mentioned above, excited structures such as $D$ will have higher energies (smaller term values) due to their charge-transfer character. The total number of such excited states (local and charge separated) in a system of $m$ atoms is $m / 2$. Thus, for ethylene, butadiene, benzene and naphthalene there are 1, 2, 3 and $5 \mathrm{C} 1 s \rightarrow n \pi^{*}$ excited states, as observed. An $m$ - 
fold unresolved degeneracy remains due to the $m$ possible $\mathrm{C} 1 s$ orbitals from which each excitation is possible. Viewed in this way, one is led to the conclusion that the resemblance between the $n \pi$ splitting pattern of naphthalene as reflected in its photoelectron spectrum and the $n \pi^{*}$ pattern as reflected in its inner-shell energy loss spectrum (Table 6) is fortuitous, for the potentials for the $n \pi$ and $n \pi^{*}$ MO's in these two cases are very different.

\section{CONCLUSIONS}

In the TNI spectra of the fluoroethylenes there is evidence for the presence of a $\sigma^{*} \mathrm{MO}$ which drops significantly in energy with increasing fluorination. However, in the Franck-Condon vertical regime, the lowest $\sigma^{*}$ MO only approaches $1 \pi^{*}$ from above and does not pass below it. In support of this, the lowest energy feature in the inner-shell spectra of all the fluoroethylenes below the relevant ionization potentials is an excitation to $1 \pi^{*}$, while the Rydberg manifold and $\mathrm{C} 1 s(\mathrm{CF}) \rightarrow \sigma^{*}$ bands occur at higher energies. Positive evidence for the validity of the perfluoro effect in the inner-shell spectral region rests in the constancy upon fluorination of the $\left(1 s, 1 \pi^{*}\right)$ term values in planar systems (aromatics, ethylene and formaldehyde) and the large increase of this term value in nonplanar systems (butyne-2, cyclopentene, acetone, acetonitrile, acetic acid) upon fluorination. Jordan and coworkers $[22,25]$ find that the $1 \pi^{*}$ energies of the ground state fluoroethylene anions are strongly dependent upon geometric factors involving not only variable $\mathrm{C}-\mathrm{C}$ and $\mathrm{C}-\mathrm{F}$ bond lengths but appreciable pyramidalization at the $\mathrm{C}$ atoms. This leads to behavior at variance with the predictions of the perfluoro effect. On the other hand, the constancy of the $1 \pi^{*}$ ionization potential in the $\left(\mathrm{C} 1 s, 1 \pi^{*}\right)$ configuration along with the strong suggestion of low-lying $\sigma^{*}$ MO's in the more heavily fluorinated species are in accord with the expectations of the perfluoro effect. We conclude that the perfluoro effect is operating on the inner-shell valence configurations when at or near the ground-state geometry. It remains an open question as to the equilibrium geometries of the fluoroethylenes in the $\left(\mathrm{C} 1 s, 1 \pi^{*}\right)$ states.

The near-constancy of the $\left(\mathrm{C} 1 s, 1 \pi^{*}\right)$ term value in a vinylogous series of compounds is surprising when viewed against the generally acknowledged depression of the $1 \pi^{*}$ MO energy with increasing extent of conjugation. It is argued that the optical electron in the $\left(\mathrm{C} 1 s, 1 \pi^{*}\right)$ excited state is strongly localized at the site of the originating $\mathrm{AO}$ and so has a term value which is independent of the extent of the neighboring groups.

As a corollary to the constancy of $\left(1 s, n \pi^{*}\right)$ term values upon perfluorination of planar systems, one also expects $\sigma^{*}$ MO's to drop significantly in energy with respect to $\pi^{*}$ MO's in these systems. Evidence is now available for such a relative stabilization in ethylene ([20] and this work) and benzene [10] following interpretation of their negative ion, vacuum UV and inner-shell spectra. 
Fluorination of unsaturated systems acts generally to increase the $\mathrm{C} 1 s \rightarrow 1 \pi^{*}$ oscillator strength by $10-100 \%$. The oscillator strengths of these valence excitations are significantly larger than those of the lower lying Rydberg excitations. $\mathrm{C} 1 s \rightarrow \sigma^{*}(\mathrm{C}-\mathrm{F})$ excitations are observed with oscillator strengths even greater than those for $\mathrm{C} 1 s \rightarrow 1 \pi^{*}$.

\section{REFERENCES}

1 M.B. Robin, Higher Excited States of Polyatomic Molecules, Vol. I, Academic Press, New York, 1974; Vol. II, Academic Press, New York, 1975; Vol. III, Academic Press, Orlando, 1985.

2 I. Ishii, R. McLaren, A.P. Hitchcock and M.B. Robin, J. Chem. Phys., 87 (1987) 4344.

3 I. Ishii, R. McLaren, A.P. Hitchcock, K.D. Jordan, H. Choi and M.B. Robin, Can. J. Chem., in press.

4 A.P. Hitchcock, D.C. Newbury, I. Ishii, J. Stöhr, J.A. Horsley, R.D. Redwing, A.L. Johnson and F. Sette, J. Chem. Phys., 85 (1986) 4849.

5 C.R. Brundle, M.B. Robin, N.A. Kuebler and H. Basch, J. Am. Chem. Soc., 94 (1972) 1451.

6 C.R. Brundle, M.B. Robin and N.A. Kuebler, J. Am. Chem. Soc., 94 (1972) 1466.

7 M.B. Yim and D.E. Wood, J. Am. Chem. Soc., 98 (1976) 2053.

8 M.B. Yim, S. DiGregori and D.E. Wood, J. Am. Chem. Soc., 99 (1973) 4260.

9 R.T. Sanderson, Polar Covalence, Academic Press, New York, 1983.

10 A.P. Hitchcock, P. Fischer, A. Gedanken and M.B. Robin, J. Phys. Chem., 91 (1987) 531.

11 T. Hayashi and T. Nakajima, Bull. Chem. Soc. Jpn., 49 (1976) 2055.

12 H. Basch, G. Bieri, E. Heilbronner and T.B. Jones, Helv. Chim. Acta, 61 (1978) 46.

13 A.P. Hitchcock, S. Beaulieu, T. Steel, J. Stöhr and F. Sette, J. Chem. Phys., 80 (1984) 3927.

14 F. Sette, J. Stöhr and A.P. Hitchcock, J. Chem. Phys., 81 (1984) 4906.

15 W.H.E. Schwarz, U. Seeger and R. Seeger, to appear in Chem. Phys.

16 H. Lefebvre-Brion and R.W. Field, Perturbations in the Spectra of Diatomic Molecules, Academic Press, New York, 1986.

17 K.D. Jordan and P.D. Burrow, Acc. Chem. Res., 11 (1978) 341.

18 H.S.W. Massey, Endeavor, 4 (1980) 78.

19 J.A. Horsley, J. Stöhr, A.P. Hitchcock, D.C. Newbury, A.L. Johnson, and F. Sette, J. Chem. Phys., 83 (1985) 6099.

20 R. McLaren, S.A.C. Clark, I. Ishii and A.P. Hitchcock, Phys. Rev. A., 36 (1987) 1683.

21 I. Ishii and A.P. Hitchcock, J. Chem. Phys., 87 (1987) 830.

22 N.S. Chiu, P.D. Burrow and K.D. Jordan, Chem. Phys. Lett., 68 (1979) 121.

23 M. Heni, E. Illenberger, H. Baumgärtel and S. Süzer, Chem. Phys. Lett., 87 (1982) 244.

24 M. Heni, G. Kwiatkowski and E. Illenberger, Ber. Bunsenges, Phys. Chem., 88 (1984) 670.

25 M.N. Paddon-Row, N.G. Rondan, K.N. Houk and K.D. Jordan, J. Am. Chem. Soc., 104 (1982) 1143.

26 P.D. Burrow, J.A. Michejda and K.D. Jordan, J. Am. Chem. Soc., 98 (1976) 6392.

27 D.F. Dance and I.C. Walker, J. Chem. Soc., Faraday Trans. 2, 71 (1975) 1903.

28 E.H. van Veen, Chem. Phys. Lett., 41 (1976) 540.

29 I.C. Walker, A. Stamatovic and S.F. Wong, J. Chem. Phys., 69 (1978) 5532.

30 J.T. Wang and F. Williams, J. Am. Chem. Soc., 103 (1981) 2902.

31 S. Bodeur, I. Nenner and P. Millie, Phys. Rev. A, 34 (1986) 2986.

32 M. Tronc, G.C. King and F.H. Read, J. Phys. B., 12 (1979) 137.

33 H.-O. Beckmann, W. Braun, H.-W. Jochims, E. Rühl and H. Baumgärtel, Chem. Phys. Lett., 121 (1985) 499. 
34 A. Barth, R.J. Buenker, S.D. Peyerimhoff and W. Butscher, Chem. Phys., 46 (1980) 149.

35 K. Seki, K. Nakagawa, N. Sato, H. Inokuchi, S. Hashimoto, K. Inoue, S. Suga, H. Kanzaki and K. Takagi, Chem. Phys. Lett., 70 (1980) 220.

36 D. Arvanitis, U. Döbler, L. Wenzel, K. Baberschke and J. Stöhr, Surf. Sci., 178 (1986) 687.

37 D. Arvanitis, K. Baberschke, L. Wenzel and U. Döbler, Phys. Rev. Lett., 57 (1986) 3175.

38 J. Stöhr, D.A. Outka, K. Baberschke, D.D. Arvanitis and J.A. Horsley, Phys. Rev. B, 36 (1987) 2976.

39 W.L. Jolly, K.D. Bomben and C.J. Eyermann, At. Data Nucl. Data Tables, 31 (1984) 433.

40 K.H. Sze, C.E. Brion and A. Katrib, to be published.

41 A.P. Hitchcock and C.E. Brion, J. Electron Spectrosc. Relat. Phenom., 13 (1978) 193; 18 (1979) 139.

42 F.C. Brown, R.Z. Bachrach and A. Bianconi, Chem. Phys. Lett., 54 (1978) 425.

43 P. Dauber and M. Brith, Chem. Phys., 11 (1975) 143.

44 A.P. Hitchcock and I. Ishii, J. Electron Spectrosc. Relat. Phenom., 42 (1987) 11.

45 A.P. Hitchcock and C.E. Brion, J. Electron Spectrosc. Relat. Phenom., 19 (1980) 231.

46 K. Vasudevan and F. Grein, Int. J. Quantum Chem., 14 (1978) 717.

47 G.R. Wight and C.E. Brion, J. Electron Spectrosc. Relat. Phenom., 4 (1974) 347.

48 C.R. Brundle and M.B. Robin, J. Am. Chem. Soc., 92 (1970) 5550.

49 G. Bieri, A. Schmelzer, L. Asbrink and M. Jonsson, Chem. Phys., 49 (1980) 213.

50 J.P. Delwiche, M-Th. Praet, G. Caprace, M.-J. Hubin-Franskin, P. Natalis and J.E. Collin, J. Electron Spectrosc. Relat. Phenom., 12 (1977) 395.

51 E. Lindholm, private communication.

52 E. Lindholm and L. Asbrink, Lecture Notes in Chemistry, Vol. 38, Springer-Verlag, Berlin, 1985.

53 P. Yannoulis, R. Dudde, K.-H. Franck and E.E. Koch, Surf. Sci., 189 (1987) 519.

54 F.A. Cotton, Chemical Applications of Group Theory, 2nd Edn., Interscience, 1971.

55 M. Bader, J. Haase, K.-H. Franck, C. Ocal and A. Puschmann, J. Phys., C8-41 (1986) 491.

56 W.H.E. Schwarz, T.C. Chang, U. Seeger and K.H. Wang, Chem. Phys., (1987) in press.

57 I. Ishii and A.P. Hitchcock, J. Electron Spectrosc. Relat. Phenom., (1987) in press.

58 D.C. Newbury and A.P. Hitchcock, unpublished.

59 A.L. Johnson, Ph.D. Thesis, University of California, Berkeley, 1986.

60 J.L. Dehmer, J. Chem. Phys., 56 (1972) 4496.

61 A.P. Hitchcock, P. Fischer and R. McLaren, in J.P. Connerade, J.M. Esteves and R.C. Karnatals (Eds), Giant Resonances in Atoms, Molecules and Solids, NATO ASI Series B, 151 (1987) 281.

62 L.C. Synder and H. Basch, Molecular Wave Functions and Properties, Wiley, New York, 1972.

63 J.N. Murrell, The Theory of the Electronic Spectra of Organic Molecules, Wiley, New York, 1963. 